\title{
InCRNA TMEM51-AS1 and RUSC1-AS1 function as ceRNAs for induction of laryngeal squamous cell carcinoma and prediction of prognosis
}

\author{
Lian Hui ${ }^{1}$, Jing Wang ${ }^{1}$, Jialiang Zhang ${ }^{1}$, Jin Long ${ }^{\text {Corresp. } 2}$ \\ ${ }^{1}$ Department of Otolaryngology, the First Hospital of China Medical University, Shenyang, Liaoning Province, China \\ 2 Department of General Surgery, the First Hospital of China Medical University, Shenyang, Liaoning Province, China \\ Corresponding Author: Jin Long \\ Email address: jinlong20187@126.com
}

Background: Long non-coding RNAs (IncRNAs) can function as competing endogenous RNAs (ceRNAs) to interact with miRNAs to regulate target genes and promote cancer initiation and progression. The expression of IncRNAs and miRNAs can be epigenetically regulated. The goal of this study was to construct an IncRNA-miRNA-mRNA ceRNA network in laryngeal squamous cell carcinoma (LSCC) and reveal their methylation patterns, which was not investigated previously.

Methods: Microarray datasets available from the Gene Expression Omnibus database were used to identify differentially expressed IncRNAs (DELs), miRNAs (DEMs), and genes (DEGs) between LSCC and controls, which were then overlapped with differentially methylated regions (DMRs). The ceRNA network was established by screening the interaction relationships between miRNAs and IncRNAs/mRNAs by corresponding databases. TCGA database was used to identify prognostic biomarkers.

Results: Five DELs (downregulated: TMEM51-AS1, SND1-IT1; upregulated: HCP5, RUSC1-AS1, LINC00324) and no DEMs were overlapped with the DMRs, but only a negative relationship occurred in the expression and methylation level of TMEM51-AS1. Five DELs could interact with 11 DEMs to regulate 242 DEGs, which was used to construct the ceRNA network, including TMEM51-AS1-miR-106b-SNX21/ TRAPPC10, LINC00324/RUSC1-AS1-miR-16-SPRY4/MICAL2/ SLC39A14, RUSC1-AS1-miR-10-SCG5 and RUSC1-AS1-miR-7-ZFP1 ceRNAs axes. Univariate Cox regression analysis showed RUSC1-AS1 and SNX21 were associated with overall survival (OS); LINC00324, miR-7 and ZFP1 correlated with recurrence-free survival (RFS); miR-16, miR-10, SCG5, SPRY4, MICAL2 and SLC39A14 were both OS and RFS-related. Furthermore, TRAPPC10 and SLC39A14 were identified as independent OS prognostic factors by multivariate Cox regression analysis.

Conclusion: DNA methylation-mediated TMEM51-AS1 and non-methylation-mediated RUSC1-AS1 may function as ceRNAs for induction of LSCC. They and their ceRNA axis genes (particularly TMEM51-AS1miR-106b-TRAPPC10; RUSC1-AS1-miR-16-SLC39A14) may be potentially important prognostic biomarkers for LSCC. 
1 IncRNA TMEM51-AS1 and RUSC1-AS1 function as ceRNAs for induction of laryngeal

\section{2 squamous cell carcinoma and prediction of prognosis}

3 Running title: ceRNAs for LSCC

4

5 Lian Hui ${ }^{1}$, Jing Wang ${ }^{1}$, Jialiang Zhang ${ }^{1}$, Jin Long $^{2}$

$6{ }^{1}$ Department of Otolaryngology, the First Hospital of China Medical University, Shenyang,

7 Liaoning Province, 110001, PR China;

8 2Department of General Surgery, the First Hospital of China Medical University, Shenyang,

9 Liaoning Province, 110001, PR China

10 Corresponding author: Jin Long.

11 Email: jinlong20187@126.com; Telephone: +86-024-83283330; Fax: +86-024-83282997.

12 Corresponding address: Department of general surgery, The First Hospital of China Medical

13 University, No.155 NanjingBei Street, Shenyang, Liaoning Province, 110001, PR China.

21 Abstract 
22 Background: Long non-coding RNAs (lncRNAs) can function as competing endogenous RNAs

23 (ceRNAs) to interact with miRNAs to regulate target genes and promote cancer initiation and

24 progression. The expression of lncRNAs and miRNAs can be epigenetically regulated. The goal

25 of this study was to construct an IncRNA-miRNA-mRNA ceRNA network in laryngeal

26 squamous cell carcinoma (LSCC) and reveal their methylation patterns, which was not

27 investigated previously.

28 Methods: Microarray datasets available from the Gene Expression Omnibus database were used

29 to identify differentially expressed lncRNAs (DELs), miRNAs (DEMs), and genes (DEGs)

30 between LSCC and controls, which were then overlapped with differentially methylated regions

31 (DMRs). The ceRNA network was established by screening the interaction relationships between

32 miRNAs and lncRNAs/mRNAs by corresponding databases. TCGA database was used to

33 identify prognostic biomarkers.

34 Results: Five DELs (downregulated: TMEM51-AS1, SND1-IT1; upregulated: HCP5, RUSC1-

35 AS1, LINC00324) and no DEMs were overlapped with the DMRs, but only a negative

36 relationship occurred in the expression and methylation level of TMEM51-AS1. Five DELs

37 could interact with 11 DEMs to regulate 242 DEGs, which was used to construct the ceRNA

38 network, including TMEM51-AS1-miR-106b-SNX21/ TRAPPC10, LINC00324/RUSC1-AS1-

39 miR-16-SPRY4/MICAL2/ SLC39A14, RUSC1-AS1-miR-10-SCG5 and RUSC1-AS1-miR-7-

40 ZFP1 ceRNAs axes. Univariate Cox regression analysis showed RUSC1-AS1 and SNX21 were

41 associated with overall survival (OS); LINC00324, miR-7 and ZFP1 correlated with recurrence-

42 free survival (RFS); miR-16, miR-10, SCG5, SPRY4, MICAL2 and SLC39A14 were both OS 
43 and RFS-related. Furthermore, TRAPPC10 and SLC39A14 were identified as independent OS

44 prognostic factors by multivariate Cox regression analysis.

45 Conclusion: DNA methylation-mediated TMEM51-AS1 and non-methylation-mediated

46 RUSC1-AS1 may function as ceRNAs for induction of LSCC. They and their ceRNA axis genes

47 (particularly TMEM51-AS1-miR-106b-TRAPPC10; RUSC1-AS1-miR-16-SLC39A14) may be

48 potentially important prognostic biomarkers for LSCC.

49

50

51

52

53

54

55

56

57

58

59

60

61

62 Introduction

63 Laryngeal squamous cell carcinoma (LSCC) is one of the common malignancies of the 
64 upper respiratory tract that has been associated with a deterioration of the environment and an

65 increase in the occupational stress. It was estimated that 13,360 new cases were diagnosed in 662017 in the United States, of which over 3,660 were fatal (Siegel et al. 2017). In China, an

67 estimated 26,400 new cases of LSCC and 14,500 cancer-related deaths also occurred in 2015

68 (Chen W 2016). Although patients with LSCC can be managed by surgical intervention,

69 radiation therapy and chemotherapy, the overall five-year survival remains poor (approximately

70 60\%) (Rudolph et al. 2011). Therefore, there is an urgent need to deeply understand the

71 molecular mechanisms underlying LSCC carcinogenesis or progression in order to develop more

72 effective therapeutic strategies.

73 Accumulating evidence has suggested that non-coding RNAs (ncRNAs) play crucial roles

74 in the initiation and development of tumors. ncRNAs are loosely categorized into small ncRNAs

75 and long non-coding RNAs (lncRNAs), both of which have regulatory functions in various

76 biological processes. The well-documented small ncRNAs are microRNAs (miRNAs; $\sim 22$

77 nucleotides long) that regulate gene expression by binding to complementary sequences in the 3'

78 untranslated region (UTR), leading to either inhibition of translation or degradation of the

79 transcripts (Jean \& Mihaela 2014). Although the mechanisms remain unclear, growing evidence

80 supports that IncRNAs could function as competing endogenous RNAs (ceRNAs) by

81 competitively binding to miRNAs through their miRNA response elements (MRE) and

82 subsequently regulate target RNA expression (Salmena et al. 2011). This ceRNA mechanism has

83 generated much interest to explain tumor development and progression in many malignancies,

84 such as gastric cancer (Song Z 2018), thyroid carcinoma (Zhao et al. 2018) and hepatoblastoma 
85 (Liu et al. 2017a). Recent studies also have preliminarily revealed several underlying ceRNA

86 regulatory interactions in LSCC. Luciferase reporter assay and Western blotting results

87 suggested that AC026166.2-001 could act as a sponge of miR-24-3p and regulate the expression

88 of p27 and cyclin D1 (Shen et al. 2018). lncRNA H19 was shown to serve as a ceRNA by

89 sponging miR-148a-3p to upregulate the target gene DNA methyltransferase 1 (Wu et al. 2016).

90 NEAT1 was also reported to regulate the expression of cyclin dependent kinase 6 through

91 modulating miR-107 (Wang et al. 2016b). Furthermore, a ceRNA network, including 30 genes,

9221 miRNAs and 19 lncRNAs was also built based on microarray analysis of 6-paired clinical

93 samples in LSCC (Zhang et al. 2016). However, analysis of the lncRNA-miRNA-mRNA

94 regulatory network of LSCC with larger sample sizes and confirmation of their clinical

95 associations are still lacking.

96 In addition, DNA methylation has been identified as an important mechanism to regulate

97 gene expression in cancer cells epigenetically, which not only regulates the expression of

98 protein-encoding genes, but also affects miRNAs and lncRNAs. For example, hyper-methylation

99 of the promoter region was observed to lead to a loss of expression of lncRNA maternally

100 expressed gene 3 (MEG3). Downregulated MEG3 was insufficient to sponge miR-9 and block its

101 inhibition effects on the expressions of E-cadherin and FOXO1, consequentially resulting in poor

102 prognosis in patients with esophageal squamous cell carcinoma (Dong et al. 2017). The study of

103 Guo et al. also suggested lncRNA CTC-276P9.1 was hyper-methylated in esophageal squamous

104 cell carcinoma. Over-expression of CTC-276P9.1 inhibited cancer cell proliferation and invasion

105 in vitro probably by regulating epithelial-mesenchymal transition (Guo W 2018). Liao et al. 
106

107 MethylCap-seq dataset (Liao et al. 2015). Cheung et al. found that the loci of three miRNAs

108 (namely miR-199a-2, miR-124a-2 and miR-184) were linked to hyper-methylated differentially

109 methylated regions (DMRs) in human testicular cancer (Cheung \& Lee 2010). However, the

110 DNA methylation regulatory mechanisms of miRNAs and lncRNAs have rarely been reported in

\section{LSCC.}

112 The goal of this study was to establish an lncRNA-miRNA-mRNA ceRNA network in

113 LSCC using larger samples and to investigate their methylation patterns. Our results may provide

114 new clues for biologists to further understand the pathogenesis of LSCC.

\section{Materials and methods}

\section{Data source}

117

lncRNA, miRNA, mRNA and methylation data were retrieved from Gene Expression

118 Omnibus (GEO) database (http://www.ncbi.nlm.nih.gov/geo/) in January 2018 according to the

119 following inclusion criteria: 1) lncRNA, miRNA, mRNA expression or methylation profiles; 2)

120 laryngeal tissue samples, not blood, interstitial fluid or cells; 3) inclusion of control; 4) human

121 samples; and 5) patients with LSCC.

122 Two lncRNA microarray datasets were obtained under accession number GSE59652 (7

123 LSCC and 7 paired adjacent normal tissues) (Shen et al. 2014) and GSE84957 (9 LSCC and 9

124 paired adjacent non-neoplastic tissues) (Feng et al. 2016). The microarray platforms of

125 GSE59652 and GSE84957 were Agilent-033010 (GPL13825, Arraystar Human LncRNA

126 microarray V2.0) and Agilent-042818 (GPL17843, Agilent-042818 Human lncRNA Micorarray

Peer) reviewing PDF | (2019:03:35639:2:0:NEW 19 Jun 2019) 
127 8_24_v2), respectively.

128 Two miRNA microarray datasets were collected under accession number GSE70289 (12

129 LSCC tissues and 4 adjacent normal tissues) (Karatas et al. 2015) and GSE62819 (5 LSCC

130 carcinoma and 5 paired adjacent non-neoplastic tissues). The microarray platforms of GSE70289

131 and GSE62819 were Agilent-031181 (GPL15018,

132 Unrestricted_Human_miRNA_V16.0_Microarray 030840) and Affymetrix Multispecies

133 miRNA-3 Array (GPL16384), respectively.

134 Four mRNA microarray datasets were available under accession number GSE51985 (10

135 LSCC and 10 paired adjacent normal tissues), GSE84957 (9 LSCC and 9 paired adjacent normal

136 tissues) (Feng et al. 2016), GSE59102 (29 LSCC and 13 normal margin tissues) and GSE58911

137 (15 LSCC and 15 normal tissue distant to LSCC) (Sharon et al. 2015). The microarray platforms

138 of GSE51985, GSE84957, GSE59102 and GSE58911 were Illumina HumanHT-12 V4.0

139 (GPL10558), Agilent-042818 (GPL17843, Human lncRNA Micorarray 8_24_v2), Agilent-

140014850 (GPL6480, Whole Human Genome Microarray 4x44K G4112F) and Affymetrix Human

141 Gene 1.0 ST Array (GPL6244), respectively.

142 One set of DNA methylation data was acquired under accession number GSE25093 (Poage

143 et al. 2012; Poage et al. 2011) which included 213 blood and 109 tissue samples. Among the 109

144 tissue samples, 56 were isolated from oral, 16 from pharyngeal, and 22 from laryngeal origin,

145 while 15 were of unclear origin. Thus, only these 22 samples from laryngeal origin (15 LSCC

146 tissues and 7 controls) were used in our study. The microarray platform of GSE25093 was

147 Illumina HumanMethylation27 BeadChip (GPL8490, HumanMethylation27_270596_v.1.2). 
148 The mRNA and miRNA Seq-data of head and neck squamous cell carcinoma (Level 3)

149 were also downloaded from The Cancer Genome Atlas (TCGA; https://tcga-data.nci.nih.gov/).

150 After sample barcode screening, 559 were miRNA-mRNA matched samples, of which 18 were

151 distributed in the alveolar crest, 30 in the root of the tongue, 22 in the buccal mucosa, 67 in the

152 mouth floor, 8 in the hard palate, 9 in the laryngeal pharynx, 138 in the larynx, 3 in the lip, 38 in

153 the oral cavity, 156 in the tongue, 10 in the oropharynx, 45 in the tonsil and 15 from an unclear

154 location. Only the 138 samples from the larynx were used in our study.

\section{Data preprocessing}

156 For the data from Affy platform, the raw data in CEL. files were preprocessed using the

157 oligo

package

(version

1.41.1;

http://www.bioconductor.org/packages/release/bioc/html/oligo.html) in $\mathrm{R}$ (version 3.4.1;

http://www.R-project.org/), including data transformation, missing value imputation with median,

background correction with MAS method and quantile normalization.

161 For the data from Agilent and Illumina platforms, the raw data in TXT. files were

162 preprocessed using the Linear Models for Microarray Data (LIMMA) package (version 3.34.0;

163 https://bioconductor.org/packages/release/bioc/html/limma.html) in $\mathrm{R}$, including data log2

164 transformation and median normalization.

165 The data (FPKM, fragment per kilobase per million mapped reads) from TCGA were

166 quantile normalized using the preprocessCore package (version 1.40.0;

167 http://bioconductor.org/packages/release/bioc/html/preprocessCore.html) in R.

\section{Differential expression analysis}


169 The differentially expressed lncRNAs (DELs) and miRNAs (DEMs) between LSCC and

170 normal controls were identified using the LIMMA method in $\mathrm{R}$ from their two included

171 microarray datasets (IncRNA: GSE59652 and GSE84957; miRNA: GSE70289 and GSE62819).

172 The $\mathrm{p}$-value $<0.05$ and $\mid \operatorname{logFC}($ fold change $) \mid>0.263$ were set as the cut-off points. The overlap

173 in the above two datasets was used for the following analysis of lncRNAs and miRNAs,

174 respectively.

175 The differentially expressed genes (DEGs) between LSCC and normal controls were

176 identified using the MetaDE.ES function in MetaDE package (version 1.0.5, https://cran.r-

177 project.org/web/packages/MetaDE/) of R from its four included microarray datasets (GSE51985,

178 GSE84957, GSE59102 and GSE58911). The p-value $<0.05$ and false discovery rate $($ FDR) $<$

1790.05 were set as the cut-off points. The DEGs with the same expression trend $\left(\operatorname{tau}^{2}\right.$ statistic $=0$,

180 p-value of Chi-square based Q-test $>0.05)$ in the four datasets were selected for the following

181 analysis.

182 Wilcoxon signed-rank test (http://127.0.0.1:26738/library/stats/html/wilcox.test.html) was

183 used to screen the DMRs between LSCC and normal controls. $\mathrm{P}<0.05$ was set as the threshold

184 value. Human annotation data were retrieved from GENCODE Release 19 (GRCh37.p13)

185 (http://www.gencodegenes.org/releases/19.html). The sequences of miRNAs, lncRNAs and

186 mRNAs in the corresponding platform GPL8490 were blasted with the GRCh37.p13 to obtain

187 the differentially methylated miRNAs, lncRNAs and mRNAs, which were then overlapped with

188 the DELs, DEMs and DEGs to screen methylated-related DELs, DEMs and DEGs, respectively.

\section{CeRNA regulatory network construction}


190 Three reliable online databases, including miRcode (version 11; http://www.mircode.org/),

191 starBase (version 2.0; http://starbase.sysu.edu.cn/index.php) (Li et al. 2014) and DIANA-

192 LncBase (version 2.0; http://carolina.imis.athena-

193 innovation.gr/diana_tools/web/index.php?r=lncbasev2/index-predicted) (Paraskevopoulou et al.

194 2013) were used to screen the interactions between lncRNAs and miRNAs. The union of these

195 three datasets was used for the following analysis. The target genes of miRNAs that were linked

196 to the lncRNAs were predicted using four frequently used algorithms, including TargetScan

197 (version 7.2; http://www.targetscan.org/vert_71/) (Agarwal V 2015), miRBase (version 22;

198 https://www.ebi.ac.uk/enright-srv/microcosm/htdocs/targets/v5/) (Griffithsjones S 2005),

199 miRanda (version 1.9; http://www.microrna.org/microrna/home.do/) (John B 2005) and

200 miRTarBase (version 7.0; http://mirtarbase.mbc.nctu.edu.tw/php/index.php) (Chou et al. 2017).

201 The target genes predicted by at least two databases and a negative association with miRNAs

202 were retained. The lncRNA-miRNA and miRNA-mRNA interactions were integrated to

203 construct the ceRNA network, which was visualized using Cytoscape software (version 3.4;

204 www.cytoscape.org/) (Kohl et al. 2011).

205 Function enrichment analysis

206 The Database for Annotation, Visualization and Integrated Discovery (DAVID) online tool

207 (version 6.8; http://david.abcc.ncifcrf.gov) (Da et al. 2009) was used for Gene Ontology (GO)

208 terms [including molecular function (MF), biological process (BP) and cellular component (CC)

209 categories] and Kyoto encyclopedia of genes and genomes (KEGG) pathway enrichment

210 analyses of genes in the ceRNA network. P-value $<0.05$ was set as the cut-off value. 


\section{Clinical associations of IncRNAs, miRNAs and mRNAs in the ceRNA network}

212 The expression levels of lncRNAs, miRNAs and mRNAs in the ceRNA network were

213 downloaded from the TCGA data. Univariate Cox regression analysis was performed to screen

214 for the prognosis-related (including overall survival, OS; and recurrence-free survival, RFS)

215 IncRNAs, miRNAs and mRNAs using the survival package (version 2.40.1; https://cran.r-

216 project.org/package=survival), which was used to construct the prognosis-related ceRNA

217 network. The samples were divided into two groups based on the expression of each lncRNA,

218 miRNA and mRNA: a low expression group ( $<$ median) and a high expression ( $>$ median) group.

219 The Kaplan-Meier method with the log-rank test was used to estimate the difference in OS and

220 RFS between the high and low expression groups. $\mathrm{P}<0.05$ was considered statistically

221 significant. Furthermore, multivariate Cox regression analysis was also performed using the

222 survival package (version 2.40.1; https://cran.r-project.org/package=survival) to evaluate the

223 prognostic independence of lncRNAs, miRNAs and mRNAs. The association of nodes in the

224 prognosis-related ceRNA network with other clinical characteristics was also analyzed using the 225

multiple linear $\quad$ regression model (https://stat.ethz.ch/R-manual/R-

226 patched/library/stats/html/lm.html) in R.

\section{Results}

\section{Differential expression analysis}

The data analysis workflow is displayed in figure 1. After data normalization (Supplemental

230 Information S1-8), the DELs, DEMs and DEGs between LSCC and normal samples were

231 screened according the stated thresholds. The results showed 306 (156 downregulated and 150 
232 upregulated) and 396 (252 downregulated and 144 upregulated) DELs were identified in the

233 datasets of GSE59652 (Figure 2A) and GSE84957 (Figure 2B) (Supplemental Information S9),

234 respectively. After comparison, 40 DELs were found to be shared in these two datasets,

235 including 6 upregulated and 20 downregulated with the consistent expression trend (Figure 3A)

236 (Supplemental Information S9); a total of 1307 (765 downregulated and 542 upregulated) and

237491 (126 downregulated and 365 upregulated) DEMs were identified in the datasets GSE62819

238 (Figure 2C) and GSE70289 (Figure 2D), respectively (Supplemental Information S9). After

239 comparison, 443 DEMs were found to be common in these two datasets, among which 152

240 upregulated and 63 downregulated DEMs were shown to have a consistent expression trend

241 (Figure 3B) (Supplemental Information S9); 2975 DEGs were found to display the similar

242 expression trend in four mRNA expression profiles GSE51985, GSE84957, GSE59102 and

243 GSE58911 (Figure 2E) (Supplemental Information S9); and 4567 DMRs were identified in the

244 LSCC genome of GSE25093 dataset, including 1616 hypomethylated and 2951 hypermethylated

245 (Figure 2F) (Supplemental Information S9). After GENCODE annotation and blast analysis, 122

246 lncRNAs, but no miRNAs were found to be located in DMRs. Subsequently, the lncRNAs and

247 mRNAs in DMRs were overlapped with their expression level data above to obtain the

248 methylation-related DELs and DEGs. Consequently, 5 DELs (TMEM51-AS1, HCP5, SND1-IT1,

249 RUSC1-AS1 and LINC00324) were screened (Figure 3C). Among these DELs, only the

250 expression and methylation levels of lncRNA TMEM51-AS1 (Figure 3D-F) were opposite,

251 indicating its expression may be regulated by methylation. These methylation-related genes were

252 used to construct the ceRNA network.

Peer] reviewing PDF | (2019:03:35639:2:0:NEW 19 Jun 2019) 


\section{CeRNA network construction}

254 Twenty-four interaction pairs between 5 DELs and 14 DEMs were predicted using miRcode,

255 starBase and DIANA-LncBase databases (Table 1). The expression trends of these DELs and

256 DEMs were opposite. Subsequently, the target genes of these 14 DEMs were predicted using

257 four algorithms, with the resultant interaction pairs of 700 in TargetScan, 486 in miRBase, 341 in

258 miRanda and 268 in miRTarBase. A total of 404 interaction pairs were ultimately left due to

259 prediction by at least two databases and a negative association between them. These interaction

260 pairs between DELs and DEMs, and between DEMs and DEGs were used to construct a ceRNA

261 network, which contained 258 nodes (5 DELs, 11 DEMs and 242 DEGs) (Figure 4). In this

262 network, TMEM51-AS1 functioned as a ceRNA to regulate SNX21 (sorting nexin family

263 member 21) and TRAPPC10 (trafficking protein particle complex 10) by sponging miR-106b;

264 LINC00324 and RUSC1-AS1 acted as ceRNAs to regulate SPRY4 (sprouty RTK signaling

265 antagonist 4), PAWR (pro-apoptotic WT1 regulator), MICAL2 (microtubule associated

266 monooxygenase, calponin and LIM domain containing 2) and SLC39A14 (solute carrier family

26739 member 14) by sponging miR-16; RUSC1-AS1 regulated SCG5 (SCG5 secretogranin V) and

268 PRDM5 (PR/SET domain 5) by competitively binding to miR-10; RUSC1-AS1 also served as

269 ceRNAs for ZFP1 (ZFP1 zinc finger protein) by binding to miR-7; HCP5 could interact with

270 miR-143 to regulate RRM2 (ribonucleotide reductase regulatory subunit M2).

\section{Function enrichment analysis}

272 The DEGs in the ceRNA network was subjected to DAVID to predict their potential

273 functions in LSCC. The results showed that 17 significant GO BP terms were enriched, including 
274 GO:0042981 regulation of apoptosis (PAWR), GO:0015031 protein transport (SNX21; SCG5),

275 cell cycle (PRDM5) and GO:0043407 negative regulation of MAP kinase activity 4 (SPRY4).

276 Six KEGG pathways were also enriched, including hsa05210:Colorectal cancer,

277 hsa04210:Apoptosis and hsa05205:Proteoglycans in cancer (Table 2).

278 Clinical associations of IncRNAs, miRNAs and mRNAs in the ceRNA network

279 In the 138 miRNA-mRNA matched samples of TCGA data, 114 had OS and 82 had RFS

280 information. Univariate Cox regression analysis in these 138 samples showed that 32 RNAs were

281 significantly associated with OS, including 1 DEL (RUSC1-AS1), 2 DEMs (hsa-miR-16 and

282 hsa-miR-10) and 29 DEGs (i.e., PAWR, SCG5, SPRY4, MICAL2, SNX21, TRAPPC10 and

283 SLC39A14); while 25 RNAs were associated with RFS, including 1 DEL (LINC00324), 3

284 DEMs (hsa-miR-16, hsa-miR-10 and hsa-miR-7) and 21 DEGs (i.e., PRDM5, SCG5, SPRY4,

285 MICAL2 and ZFP1) (Table 3). The OS and RFS related ceRNA networks were extracted

286 independently as shown in Figure 5A and B.

287 Subsequently, multivariate Cox regression showed TRAPPC10 and SLC39A14 were

288 independent factors for OS; RRM2 was an independent factor for RFS (Table 4). Although

289 SOD2, SLC44A1 and THEM4 were also screened to be significant, their hazard ratios (HR)

290 were not consistent with the expected according to their expression levels. Combined with the

291 univariate results, we suggested TRAPPC10 and SLC39A14 related ceRNA axes (TMEM51-

292 AS1-miR-106-TRAPPC10; RUSC1-AS1-miR-16-SLC39A14) may be especially important. The

293 Kaplan-Meier curve of these lncRNAs, miRNAs and mRNAs were drawn. As expected, the low

294 expression of miR-16 (Figure 5D) was associated with poor prognosis and the high expression of 
295

296

297

298

299

300

301

302

303

304

305

306

307

308

309

310

311

312

313

314 TMEM51-AS1 was significantly correlated with poor OS in chromophobe renal cell carcinoma

315 (He et al. 2016). In present study, we predicted that TMEM51-AS1 might function as a ceRNA 
316 to regulate SNX21 and TRAPPC10 through sponging miR-106b. Evidence demonstrated that

317 miR-106b was up-regulated in LSCC (Lu et al. 2014; Xing et al. 2014), which was also

318 confirmed in our microarray study. miR-106b was reported to promote the proliferation and

319 invasion of LSCC cells by targeting RUNX3 (Ying et al. 2013), while induce cell cycle G0/G1

320 arrest by inhibiting tumor suppressor RB (Cai et al. 2011). Although no study revealed the roles

321 of SNX21 in cancer, its family genes, such as SNX1 (Zhan et al. 2018), SNX5 (Jitsukawa et al.

322 2017) and SNX9 (Bendris et al. 2016) were suggested to be tumor suppressor related. Therefore,

323 SNX21 may be theoretically downregulated in LSCC by miR-106b. Consistent with this

324 hypothesis, our study showed that SNX21 was less expressed in LSCC tissues and patients with

325 high expression of SNX21 had a higher OS rate. There was only one study to suggest the roles of

326 TRAPPC10 until now and showed TRAPPC10 was an oncogenic driver to predict the poor

327 prognosis for breast cancer patients (Pongor et al. 2015), which seemed to be contrast with our

328 results, implying TRAPPC10 may be a new tumor suppressor gene for LSCC. The tumor

329 inhibition effects of TRAPPC10 may be related with its potential to activate GTPase RAB11

330 (Milev et al. 2018) and then Rab coupling protein, targeted deletion of which led to accelerated

331 tumor onset (Boulay et al. 2016).

332 Furthermore, we identified several other ceRNA axes, although they were not methylation-

333 related, including LINC00324/RUSC1-AS1-miR-16-SPRY4/MICAL2/ SLC39A14, RUSC1-

334 AS1-miR-10-SCG5 and RUSC1-AS1-miR-7-ZFP1. All these lncRNAs, miRNAs and mRNAs

335 were significantly associated with OS and/or RFS, indicating these ceRNA axes may also be 336 underlying therapeutic targets. 

confirmed in LSCC samples. Accumulating evidence also has proved the roles of miR-16, miR-7 and miR-10 in various types of cancer. miR-16 could be downregulated in tissue samples and

341 cell lines of lung cancer (Ke Y 2013) and osteosarcoma (Jiao et al. 2018). Ectopic expression of

342 miR-16 inhibited cell proliferation, colony formation in vivo and, migration and invasion in vitro

343 by regulating its target genes RAB23 and Smad3 (Jiao et al. 2018; Zhang et al. 2018). miR-10a

344 was down-regulated in laryngeal epithelial premalignant lesions with increasing grade of

345 dysplasia (Hu et al. 2015). Overexpression of miR-10a inhibited cell metastasis by regulating

346 epithelial-to-mesenchymal transition (EMT) (Liu et al. 2017b). miR-7-5p was lower expressed in

347 brain-metastatic lesions of breast cancer (Hiroshi et al. 2013) and the use of miR-7-5p mimics

348 suppressed cell proliferation and induced apoptosis (Shi et al. 2015) via modulating the

349 expression of Kruppel like factor 4. In agreement with these studies, we also found that these

350 three miRNAs were less expressed (especially miR-10 and miR-7) in LSCC and negatively

351 associated with OS and/or RFS. Although the downstream target genes of these miRNAs have

352 been reported as above, their functions in LSCC remain poorly understood. We predicted that

353 SPRY4/MICAL2/SLC39A14, SCG5 and ZFP1 may be the potential targets of miR-16, miR-10

354 and miR-7, respectively in LSCC, which had not been validated previously. Nevertheless, the

355 studies on the molecular mechanisms of these DEGs may indirectly explain their potential

356 interactions. The expression of SPRY4 was upregulated in testicular germ cell tumors (Tian et al.

357 2018). MICAL2 was a recently identified proto-oncogene, which increased cell proliferation to 
358

359

360

361

362

363

364

365

366

367

368

369

370

371

372

373

374

375

376

377

378 accelerate tumor growth, and promoted the expression of EMT-related proteins to increase cell metastasis (Mariotti et al. 2016; Wang et al. 2018). Immunohistochemical analysis showed the expression level of SLC39A14 was significantly higher in hepatocellular carcinoma tissues than that in adjacent tissues and negatively correlated with survival time (Gartmann et al. 2018). Also, the upregulation of SLC39A14 in tumor cells may be attributed to the loss of its interactive gene p53, a tumor suppressor (Zhao et al. 2017). Although there were no studies to discuss the roles of SCG5 in cancer, its family member secretogranin II and III had been seen to be overexpressed in prostate cancer (Courel et al. 2014) and small cell lung carcinoma (Togayachi et al. 2017), suggesting SCG5 may also be oncogenic for LSCC. Zinc finger proteins had also been observed to promote cell growth and metastasis in nasopharyngeal carcinoma ( $\mathrm{Li}$ et al. 2015). In line with these findings, SPRY4, MICAL2, SLC39A14, SCG5 and ZFP1 were all upregulated in LSCC and associated with poor prognosis.

There were some limitations in this study. First, although all the known microarray or sequencing data from the public database had been included, the sample size was still not large which may influence the results. Therefore, additional clinical trials with larger samples may be essential to confirm their expression and prognosis. Second, we only preliminarily predicted that these ceRNA axes may be associated with LSCC development and prognosis. The regulatory relationships between lncRNAs and miRNAs as well as between miRNAs and mRNAs needed further experimental confirmation in vitro and in vivo (i.e., dual luciferase reporter assay or lossof-function). Third, whether the expression of TMEM51-AS1 was regulated by methylation should be validated by using the methylation inhibitor 5-azacytidine. Fourth, although we have

Peer) reviewing PDF | (2019:03:35639:2:0:NEW 19 Jun 2019) 
379 normalized the data from different platforms, this may still cause some underlying bias.

380 Conclusion

381 Our present study identifies several important mechanisms for the development and

382 progression of LSCC: 1) methylation-mediated upregulation of lncRNA TMEM51-AS1 may

383 function as a ceRNA for miR-106b to regulate SNX21 and TRAPPC10; 2) survival-related

384 RUSC1-AS1/LINC00324 may function as a ceRNA to sponge miR-16, miR-10 or miR-7 and

385 then regulate SPRY4/ MICAL2/SLC39A14, SCG5/PRDM5 and ZFP1, respectively. Altogether,

386 these IncRNA, miRNAs or mRNAs may be potential prognostic biomarkers and therapeutic

387 targets of LSCC.

388 Acknowledgements

$389 \quad$ None.

390 References

391 Agarwal V, Bell KW, Nam JW, Bartel DP. 2015. Predicting effective microRNA target sites in 392 mammalian mRNAs. Elife 4: e05005.

393 Bendris N, Stearns CJS, Reis CR, Rodriguezcanales J, Liu H, Witkiewicz AW, and Schmid SL.

394 2016. Sorting nexin 9 negatively regulates invadopodia formation and function in cancer $395 \quad$ cells. J Cell Sci 129:2804-2816.

396 Boulay PL, Mitchell L, Turpin J, Huot-Marchand JÉ, Lavoie C, Sanguin-Gendreau V, Jones L,

397 Mitra S, Livingstone JM, Campbell S, Hallett M, Mills GB, Park M, Chodosh L,

398 Strathdee D, Norman JC, Muller WJ. 2016. Rab11-FIP1C Is a Critical Negative

399 Regulator in ErbB2-Mediated Mammary Tumor Progression. Cancer Res 76:2662-2674. 
400 Cai K, Wang Y, and Bao X. 2011. MiR-106b promotes cell proliferation via targeting RB in $401 \quad$ laryngeal carcinoma. J Exp Clin Cancer Res 30:73.

402 Chen W ZR, Baade PD, Zhang S, Zeng H, Bray F, Jemal A, Yu XQ, He J. 2016. Cancer 403 statistics in China, 2015. Ca Cancer J Clin 66:115-132.

404

Cheung HH, and Lee TA. 2010. Genome-wide DNA methylation profiling reveals novel 405 epigenetically regulated genes and non-coding RNAs in human testicular cancer. $\mathrm{Br} J$ Cancer 102:419-427.

407

Chou CH, Shrestha S, Yang CD, Chang NW, Lin YL, Liao KW, Huang WC, Sun TH, Tu SJ, 408 and Lee WH. 2017. miRTarBase update 2018: a resource for experimentally validated microRNA-target interactions. Nucleic Acids Res 46: D296-D302.

410 Courel M, El Yamani FZ, Alexandre D, El FH, Delestre C, Montero-Hadjadje M, Tazi F, Amarti A, Magoul R, Chartrel N. 2014. Secretogranin II is overexpressed in advanced prostate cancer and promotes the neuroendocrine differentiation of prostate cancer cells. Eur $J$ Cancer 50:3039-3049.

Da WH, Sherman BT, and Lempicki RA. 2009. Systematic and integrative analysis of large gene lists using DAVID bioinformatics resources. Nat Protoc 4:44-57.

416 Dong Z, Zhang A, Liu S, Lu F, Guo Y, Zhang G, Xu F, Shi Y, Shen S, and Liang J. 2017. Aberrant Methylation-Mediated Silencing of lncRNA MEG3 Functions as a ceRNA in Esophageal Cancer. Mol Cancer Res 15:800-810.

419 Feng L, Wang R, Lian M, Ma H, He N, Liu H, Wang H, and Fang J. 2016. Integrated Analysis of Long Noncoding RNA and mRNA Expression Profile in Advanced Laryngeal 
Squamous Cell Carcinoma. Plos One 11:e0169232.

422 Gartmann L, Wex T, Grüngreiff K, Reinhold D, Kalinski T, Malfertheiner P, Schütte K. 2018.

423 Expression of zinc transporters ZIP4, ZIP14 and ZnT9 in hepatic carcinogenesis-An

424 immunohistochemical study. J Trace Elem Med Biol 49:35-42.

425 Griffithsjones S GR, Van DS, Bateman A, Enright AJ. 2005. miRBase: microRNA sequences, $426 \quad$ targets and gene nomenclature. Nucleic Acids Res 34:140-144.

427 Guo W, Liu S, Dong Z, Guo Y, Ding C, Shen S, Liang J, Shan B. 2018. Aberrant methylation428 mediated silencing of lncRNA CTC-276P9.1 is associated with malignant progression of esophageal squamous cell carcinoma. Clin Exp Metastasis 35:53-68.

430 Guo W, Lv P, Liu S, Xu F, Guo Y, Shen S, Liang J, Kuang G, and Dong Z. 2018. Aberrant methylation-mediated downregulation of long noncoding RNA C5orf66-AS1 promotes the development of gastric cardia adenocarcinoma. Mol Carcinog 57:854-865.

433 He HT, Xu M, Kuang Y, Han XY, Wang MQ, and Yang Q. 2016. Biomarker and competing endogenous RNA potential of tumor-specific long noncoding RNA in chromophobe renal cell carcinoma. Onco Targets Ther 9:6399-6406.

436 Hiroshi O, Fei X, Pandey PR, Sambad S, Misako W, Pai SK, Yin-Yuan M, Megumi IG, Shigeru H, and Yin L. 2013. miR-7 suppresses brain metastasis of breast cancer stem-like cells by modulating KLF4. Cancer Res 73:1434-1444.

Hu Y, Jin Y, Li X, Yu J, Wang F, Bu W, and Liu H. 2015. [Expression of microRNA-10a-5p in laryngeal epithelial premalignant lesions]. Zhonghua Bing LI Xue Za Zhi 44:184-188.

441 Jean H, and Mihaela Z. 2014. Identification and consequences of miRNA-target interactions-- 
beyond repression of gene expression. Nat Rev Genet 15:599-612.

443 Jian Z, Dahua F, Zhixiang J, Chen GG, and Lai PBS. 2015. Cancer Specific Long Noncoding

444 RNAs Show Differential Expression Patterns and Competing Endogenous RNA Potential

445 in Hepatocellular Carcinoma. Plos One 10:e0141042.

446 Jiao ZH, Wang JD, and Wang XJ. 2018. MicroRNA-16 suppressed the invasion and migration of 447 osteosarcoma by directly inhibiting RAB23. Eur Rev Med Pharmacol Sci 22:2598-2605.

448 Jitsukawa S, Kamekura R, Kawata K, Ito F, Sato A, Matsumiya H, Nagaya T, Yamashita K, 449 Kubo T, and Kikuchi T. 2017. Loss of sorting nexin 5 stabilizes internalized growth 450 factor receptors to promote thyroid cancer progression. J Pathol 243:342-353.

451 John B, Enright A, Aravin A, Tuschl T, Sander C. 2005. Human MicroRNA targets. PLoS Biol 452 3:e264.

453

Karatas OF, Suer I, Yuceturk B, Yilmaz M, Hajiyev Y, Creighton CJ, Ittmann M, and Ozen M. 2015. The role of miR-145 in stem cell characteristics of human laryngeal squamous cell carcinoma Hep-2 cells. Tumour Biology 37: 4183-4192.

Ke Y, Zhao W, Xiong J, Cao R. 2013. Downregulation of miR-16 promotes growth and motility by targeting HDGF in non-small cell lung cancer cells. FEBS Lett 587:3153-3157.

Kohl M, Wiese S, and Warscheid B. 2011. Cytoscape: software for visualization and analysis of biological networks. Methods Mol Biol 696:291-303.

Li JH, Liu S, Zhou H, Qu LH, Yang JH. 2014. starBase v2.0: decoding miRNA-ceRNA, miRNA-ncRNA and protein-RNA interaction networks from large-scale CLIP-Seq data. Nucleic Acids Res 42:D92-97. 
463 Li Y, Yan X, Yan L, Shan Z, Liu S, Chen X, Zou J, Zhang W, and Jin Z. 2015. High expression

464 of Zinc-finger protein X-linked is associated with reduced E-cadherin expression and

465 unfavorable prognosis in nasopharyngeal carcinoma. International Int J Clin Exp Pathol

$466 \quad 8: 3919-3927$.

467 Liao Q, He W, Liu J, Cen Y, Luo L, Yu C, Li Y, Chen S, and Duan S. 2015. Identification and

468 functional annotation of lncRNA genes with hypermethylation in colorectal cancer. Gene

469 572:259-265.

470

471

472

473

474

475

476

477

478

479

480

481

482

483

Liu S, Xie F, Xiang X, Liu S, Dong S, Qu K, and Lin T. 2017a. Identification of differentially expressed genes, lncRNAs and miRNAs which are associated with tumor malignant phenotypes in hepatoblastoma patients. Oncotarget 8:97554-97564.

Liu Y, Zhang Y, Wu H, Li Y, Zhang Y, Liu M, Li X, and Tang H. 2017b. miR-10a suppresses colorectal cancer metastasis by modulating the epithelial-to-mesenchymal transition and anoikis. Cell Death Dis 8:e2739.

Lu ZM, Lin YF, Jiang L, Chen LS, Luo XN, Song XH, Chen SH, and Zhang SY. 2014. Microribonucleic acid expression profiling and bioinformatic target gene analyses in laryngeal carcinoma. Onco Targets Ther 2014:525-533.

Mariotti S, Barravecchia I, Vindigni C, Pucci A, Balsamo M, Libro R, Senchenko V, Dmitriev A, Jacchetti E, and Cecchini M. 2016. MICAL2 is a novel human cancer gene controlling mesenchymal to epithelial transition involved in cancer growth and invasion. Oncotarget 7:1808-1825.

Milev MP, Graziano C, Karall D, Kuper WFE, Al-Deri N, Cordelli DM, Haack TB, Danhauser 

allelic mutations in TRAPPC2L result in a neurodevelopmental disorder and have an impact on RAB11 in fibroblasts. J Med Genet 55:753-764.

Militello G, Weirick T, John D, Döring C, Dimmeler S, and Uchida S. 2017. Screening and validation of lncRNAs and circRNAs as miRNA sponges. Brief Bioinform 18:780-788.

Paraskevopoulou MD, Georgios G, Nikos K, Martin R, Manolis M, Dalamagas TM, and Hatzigeorgiou AG. 2013. DIANA-LncBase: experimentally verified and computationally predicted microRNA targets on long non-coding RNAs. Nucleic Acids Res 41:D239-245.

Poage GM, Butler RA, E Andrés H, Mcclean MD, Nelson HH, Christensen BC, Marsit CJ, and Kelsey KT. 2012. Identification of an epigenetic profile classifier that is associated with survival in head and neck cancer. Cancer Res 72:2728-2737.

Poage GM, E Andres H, Christensen BC, Butler RA, Michele AW, Mcclean MD, Tim W, Michael P, Marsit CJ, and Kelsey KT. 2011. Global hypomethylation identifies Loci targeted for hypermethylation in head and neck cancer. Clin Cancer Res 17:3579-3589.

Pongor L, Kormos M, Hatzis C, Pusztai L, Szabó A, Győrffy B. 2015. A genome-wide approach to link genotype to clinical outcome by utilizing next generation sequencing and gene chip data of 6,697 breast cancer patients. Genome Med 7:104. a meta-analysis. Eur Arch Otorhinolaryngol 268:165-179. 
505 Salmena L, Poliseno L, Tay Y, Kats L, and Pandolfi PP. 2011. ceRNA hypothesis: The Rosetta

$506 \quad$ Stone of a hidden RNA language? Cell 146:353-358.

507 Sharon L, Mary E G, Robert D H, Karen T P, Michael R G, Chindo H, and Tejaswi K. 2015.

508 Prognostic biomarkers for HNSCC using quantitative real-time PCR and microarray

509 analysis: $\beta$-tubulin isotypes and the p53 interactome. Cytoskeleton 71:628-637.

510 Shen Z, Hao W, Zhou C, Deng H, Dong Y, Li Q, Lin L, Bing C, and Guo J. 2018. Long non-

511 coding RNA AC026166.2-001 inhibits cell proliferation and migration in laryngeal

512 squamous cell carcinoma by regulating the miR-24-3p/p27 axis. Sci Rep 8:3375.

513 Shen Z, Li Q, Deng H, Lu D, Song H, and Guo J. 2014. Long Non-Coding RNA Profiling in

514 Laryngeal Squamous Cell Carcinoma and Its Clinical Significance: Potential Biomarkers

515 for LSCC. Plos One 9:e108237.

516 Shi Y, Luo X, Li P, Tan J, Wang X, Xiang T, and Ren G. 2015. miR-7-5p suppresses cell

517 proliferation and induces apoptosis of breast cancer cells mainly by targeting REG $\gamma$.

$518 \quad$ Cancer Lett 358:27-36.

519 Siegel RL, Miller KD, and Jemal A. 2017. Cancer Statistics, 2017. Ca Cancer J Clin 67:7-30.

520 Song Z ZW, Cao D, Zhang J, Chen S. 2018. Elementary screening of lymph node metastatic-

521 related genes in gastric cancer based on the co-expression network of messenger RNA,

522 microRNA and long non-coding RNA. Braz J Med Biol Res 51:e6685.

523 Tian Y, Fu X, Li Q, Wang Y, Fan D, Zhou Q, Kuang W, and Shen L. 2018. MicroRNA-181

524 serves an oncogenic role in breast cancer via the inhibition of SPRY4. Mol Med Rep

$525 \quad 18: 5603-5613$. 
526 Togayachi A, Iwaki J, Kaji H, Matsuzaki H, and Narimatsu H. 2017. Glycobiomarker,

527 fucosylated short-form secretogranin III levels are increased in the serum of patients with

528 small cell lung carcinoma. J Proteome Res 16:4495-4505.

529 Wang P, Wu T, Zhou H, Jin Q, He G, Yu H, Xuan L, Wang X, Tian L, and Sun Y. 2016. Long

530 noncoding RNA NEAT1 promotes laryngeal squamous cell cancer through regulating

531 miR-107/CDK6 pathway. J Exp Clin Cancer Res 35:22.

532 Wang Y, Deng W, Zhang Y, Sun S, Zhao S, Chen Y, Zhao X, Liu L, and Du J. 2018. MICAL2

533 promotes breast cancer cell migration by maintaining epidermal growth factor receptor

534 (EGFR) stability and EGFR/P38 signalling activation. Acta Physiologica 222:e12920.

535 Wu T, Qu L, He G, Tian L, Li L, Zhou H, Jin Q, Ren J, Wang Y, and Wang J. 2016. Regulation

536 of laryngeal squamous cell cancer progression by the lncRNA H19/miR-148a-

537 3p/DNMT1 axis. Oncotarget 7:11553-11566.

538 Xing Y, Yibo W, Yang L, Hongxia D, Zhisen S, Bingxiu X, and Junming G. 2014. miR-21,

539 miR-106b and miR-375 as novel potential biomarkers for laryngeal squamous cell

$540 \quad$ carcinoma. Curr Pharm Biotechnol 15:503-508.

541 Ying X, Kai W, Wei G, Zhang C, Huang F, Wen S, and Wang B. 2013. MicroRNA-106b

542 regulates the tumor suppressor RUNX3 in laryngeal carcinoma cells. FEBS Lett $587: 3166-3174$

544 Zhan XY, Zhang Y, Zhai E, Zhu QY, and He Y. 2018. Sorting nexin-1 is a candidate tumor suppressor and potential prognostic marker in gastric cancer. Peer J 6:e4829.

546 Zhang C, Gao W, Wen S, Wu Y, Fu R, Zhao D, Chen X, and Wang B. 2016. Potential key 
molecular correlations in laryngeal squamous cell carcinoma revealed by integrated analysis of mRNA, miRNA and lncRNA microarray profiles. Neoplasma 63:888-900.

549 Zhang H, Yang K, Ren T, Huang Y, Tang X, and Guo W. 2018. miR-16-5p inhibits chordoma cell proliferation, invasion and metastasis by targeting Smad3. Cell Death Dis 9:680.

551

559

560

561

562

563

564

565

566

567

58

\section{2}

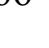

Zhao Y, Wang H, Wu C, Yan M, Wu H, Wang J, Yang X, and Shao Q. 2018. Construction and investigation of lncRNA-associated ceRNA regulatory network in papillary thyroid cancer. Oncol Rep 39:1197-1206.

Zhao N, Zhang AS, Wortham AM, Jue S, Knutson MD, Enns CA. 2017. The Tumor Suppressor, P53, Decreases the Metal Transporter, ZIP14. Nutrients 9: pii: E1335.

Zhou Z, Lin Z, Xin P, Tariq MA, Xiang A, Li P, and Wang J. 2018. Epigenetic regulation of long non-coding RNAs in gastric cancer. Oncotarget 9:19443-19458. 
571 Figure legends:

572 Figure 1 The data analysis workflow.

573 Figure 2 Hierarchical clustering and heat map analysis. A-B, heat map for differentially

574 expressed lncRNAs identified in GSE59652 (A) and GSE84957 (B) datasets; C-D, heat map for

575 differentially expressed miRNAs identified in GSE62819 (C) and GSE70289 (D) datasets; E,

576 heat map for differentially expressed genes identified by meta-analysis of GSE51985, GSE84957,

577 GSE59102 and GSE58911 datasets; F, heat map for differentially methylated regions identified

578 in GSE25093 dataset. The datasets of laryngeal squamous cell carcinoma collected from Gene

579 Expression Omnibus database. Red, high expression (hyper-methylation); green, low expression

580 (hypo-methylation).

581 Figure 3 Overlapped genes identification. Venn diagram drawing to display the overlap of

582 differentially expressed lncRNAs (A) and miRNAs (B) in different datasets of laryngeal

583 squamous cell carcinoma collected from Gene Expression Omnibus database and their overlap

584 with differentially methylated regions $(\mathrm{C})$ to screen methylation related lncRNAs and miRNAs.

585 The expression (D-E) and methylated (F) levels of overlapped lncRNAs are displayed in a

586 histogram. $^{*} \mathrm{p}<0.05 ; * * \mathrm{p}<0.01$. Contra-regulated: the expression trend of lncRNAs or

587 miRNAs was different in two datasets. Up regulated or down regulated: lncRNAs or miRNAs

588 exhibited the similar expression trend in two datasets, high or down expressed. 
589 Figure 4 Competing endogenous RNAs (ceRNAs) interaction network of lncRNA-miRNA-

590 mRNA in laryngeal squamous cell carcinoma. A, interaction pairs among upregulated lncRNAs,

591 downregulated miRNAs and upregulated mRNAs; B, interaction pairs among downregulated

592 lncRNAs, upregulated miRNAs and downregulated mRNAs. Square nodes represent lncRNAs;

593 triangle nodes represent miRNAs; round nodes represent mRNAs. Edges represent the possible

594 associations between lncRNAs, miRNAs and mRNAs. Red, upregulated; green, downregulated.

595 The red line, the interaction between lncRNAs and miRNAs; the greyish line, the interaction

596 between miRNA and mRNAs.

597 Figure 5 Prognosis related competing endogenous RNAs (ceRNAs) interaction axes. A,

598 IncRNA-miRNA-mRNA network for overall survival; B, lncRNA-miRNA-mRNA network for

599 recurrence free survival. Square nodes represent lncRNAs; triangle nodes represent miRNAs;

600 round nodes represent mRNAs. Edges represent the possible associations between lncRNAs,

601 miRNAs and mRNAs. Red, upregulated; green, downregulated.; Kaplan-Meier analysis of the

602 IncRNA (C), miRNA (D) and mRNA (E) of crucial ceRNA axis in which all lncRNA, miRNA

603 and mRNA were prognosis-related and mRNA was an independent prognostic factor.

604 Figure 6 Kaplan-Meier curve of lncRNA TMEM51-AS1 ceRNA related mRNAs.

605 A, TRAPPC10, which was an independent prognostic factor; B, SNX21, which was overall

606 survival related in univariate Cox regression analysis.

607 Supplementary Materials:

608 Supplemental Information S1: Raw data of GSE59652.

609 Supplemental Information S2: Raw data of GSE84957. 
610 Supplemental Information S3: Raw data of GSE62819.

611 Supplemental Information S4: Raw data of GSE70289.

612 Supplemental Information S5: Raw data of GSE51985.

613 Supplemental Information S6: Raw data of GSE58911.

614 Supplemental Information S7: Raw data of GSE59102.

615 Supplemental Information S8: Raw data of GSE84957.

616 Supplemental Information S9: Differentially expressed lncRNAs, miRNAs, genes and

617 differentially methylated regions in all datasets.

618 Figure S1: Kaplan-Meier curve of the lncRNA, miRNAs and mRNAs for overall survival related

619 competing endogenous RNAs interaction axes.

620 Figure S2: Kaplan-Meier curve of the lncRNA, miRNAs and mRNAs for recurrence free

621 survival related competing endogenous RNAs interaction axes. 
Figure 1

\section{The data analysis workflow}

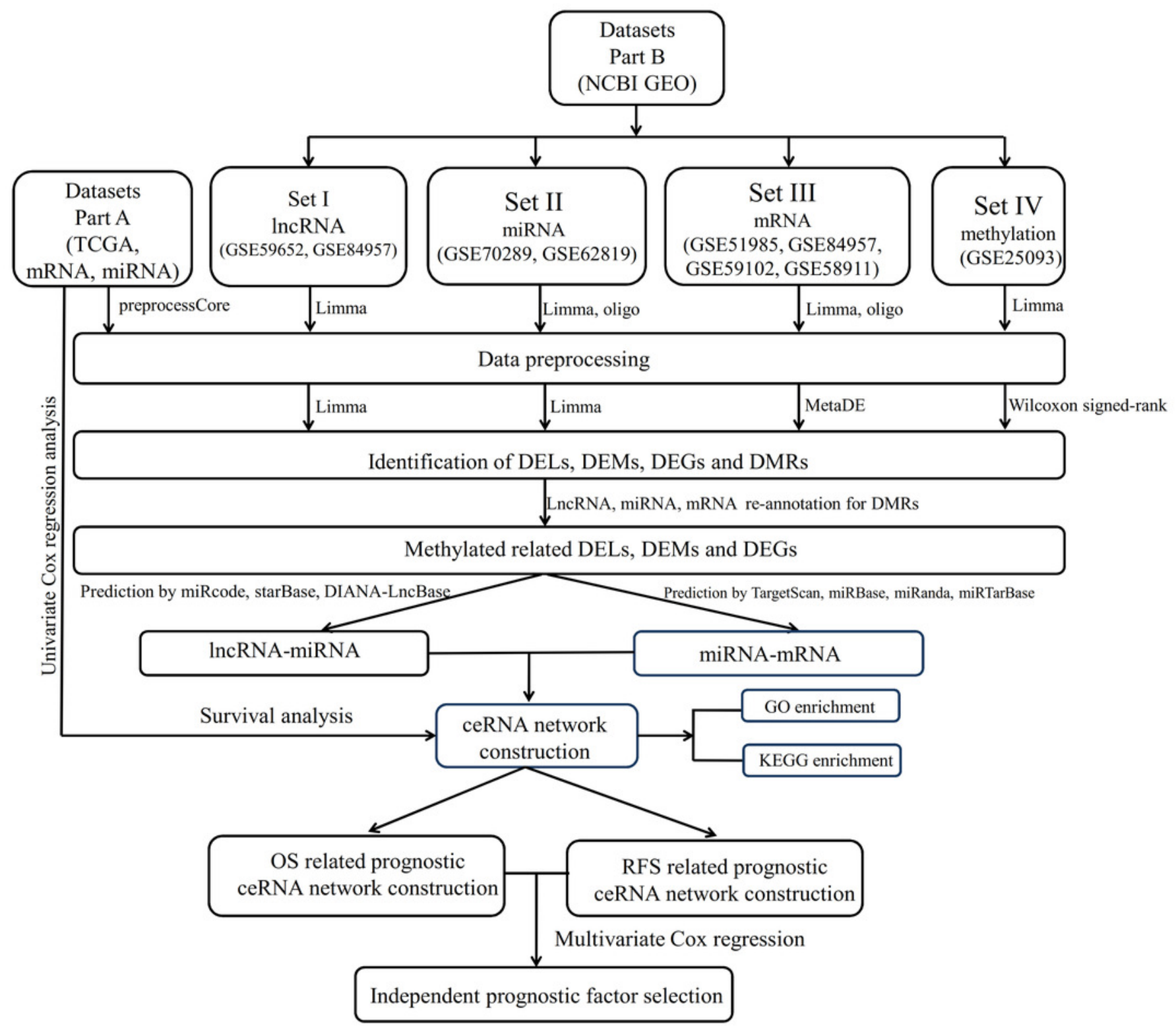




\section{Figure 2}

Hierarchical clustering and heat map analysis.

A-B, heat map for differentially expressed IncRNAs identified in GSE59652 (A) and GSE84957

(B) datasets; C-D, heat map for differentially expressed miRNAs identified in GSE62819 (C) and GSE70289 (D) datasets; E, heat map for differentially expressed genes identified by meta-analysis of GSE51985, GSE84957, GSE59102 and GSE58911 datasets; F, heat map for differentially methylated regions identified in GSE25093 dataset. The datasets of laryngeal squamous cell carcinoma collected from Gene Expression Omnibus database. Red, high expression (hyper-methylation); green, low expression (hypo-methylation).

A

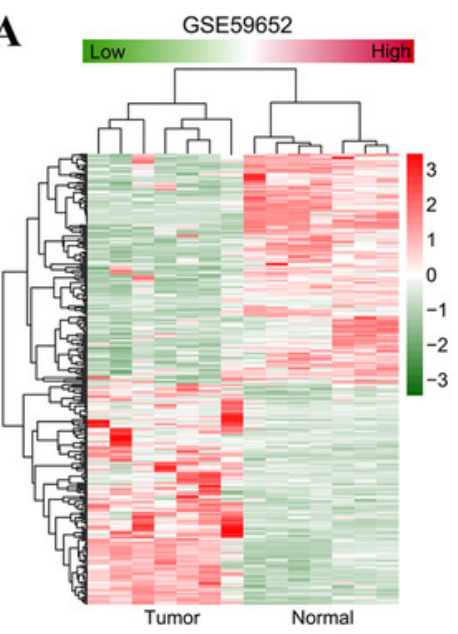

C

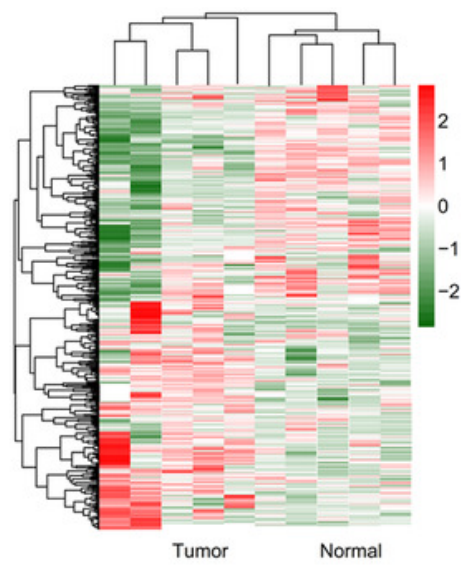

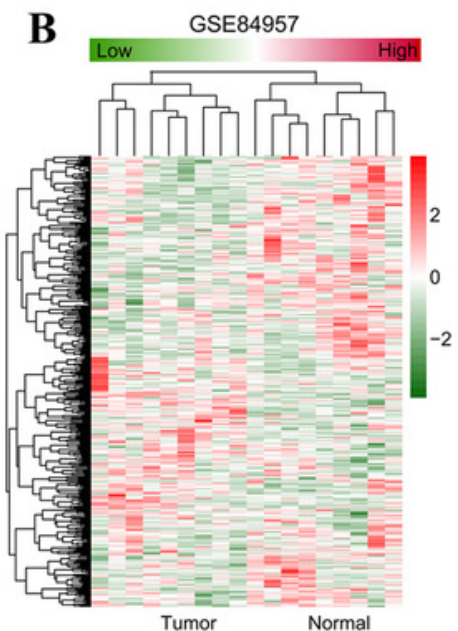

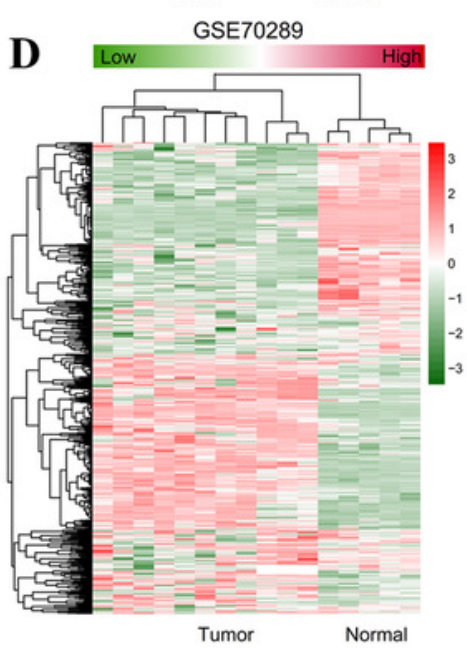

$\mathbf{E}$

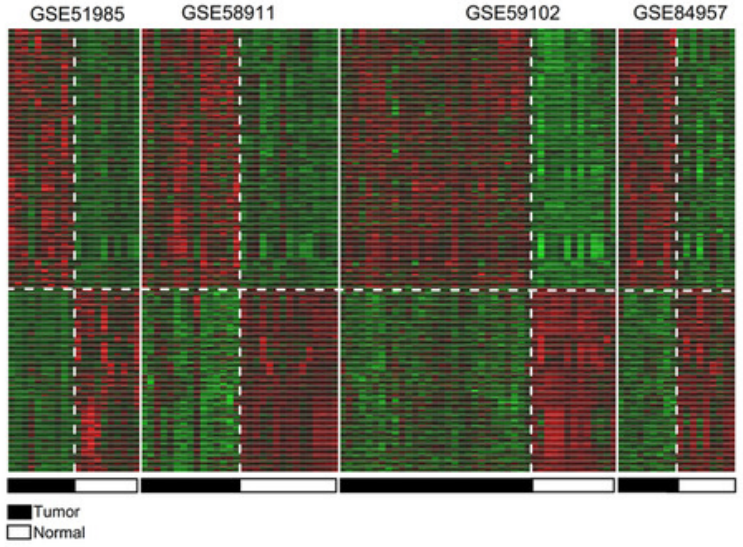

F

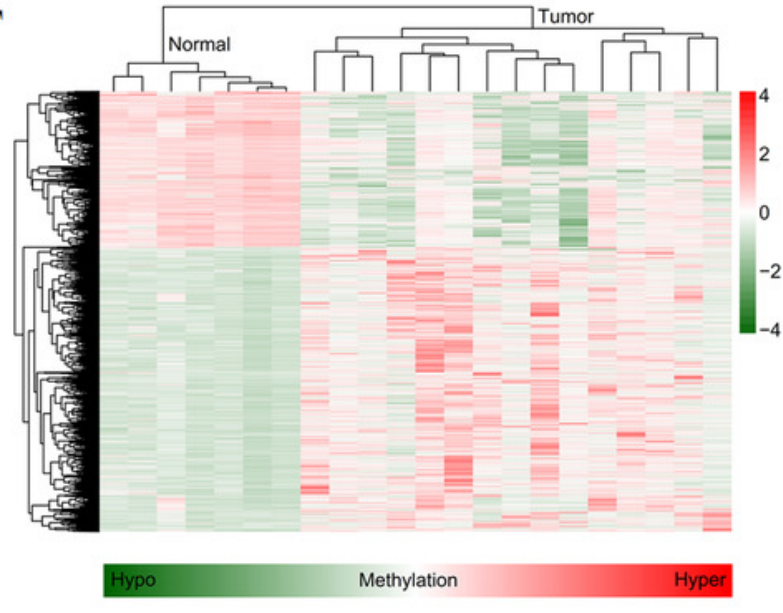




\section{Figure 3}

Overlapped genes identification.

Venn diagram drawing to display the overlap of differentially expressed IncRNAs (A) and miRNAs (B) in different datasets of laryngeal squamous cell carcinoma collected from Gene Expression Omnibus database and their overlap with differentially methylated regions (C) to screen methylation related IncRNAs and miRNAs. The expression (D-E) and methylated (F) levels of overlapped IncRNAs are displayed in a histogram.* $p<0.05$; $* *<0.01$. Contraregulated: the expression trend of IncRNAs or miRNAs was different in two datasets. Up regulated or down regulated: IncRNAs or miRNAs exhibited the similar expression trend in two datasets, high or down expressed.

$\mathbf{A}$

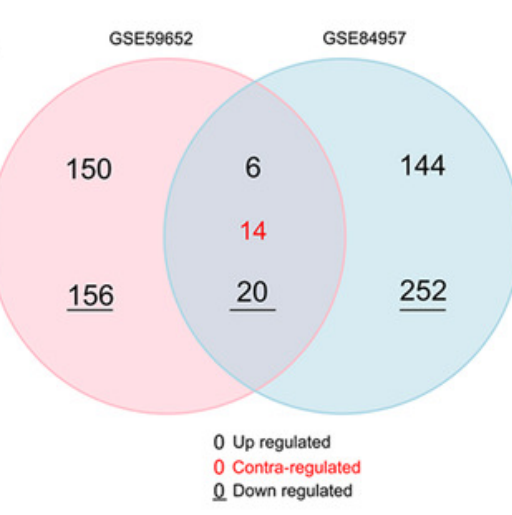

D

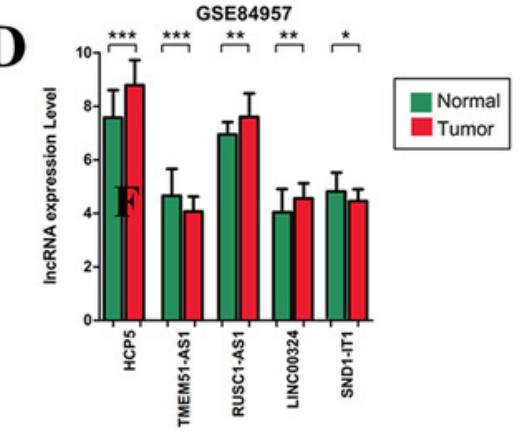

B

GSE62819

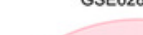

365

126

0 Up regulated

0 Contra-regulate

o Down regulated
GSE70289

542

228

63
C

Expression Level

782

2683 IncRNA 5
mRNA 777

$\underline{765}$ 


\section{Figure 4}

Competing endogenous RNAs (ceRNAs) interaction network of IncRNA-miRNA-mRNA in laryngeal squamous cell carcinoma.

A, interaction pairs among upregulated InCRNAs, downregulated miRNAs and upregulated mRNAs; B, interaction pairs among downregulated IncRNAs, upregulated miRNAs and downregulated mRNAs. Square nodes represent IncRNAs; triangle nodes represent miRNAs; round nodes represent mRNAs. Edges represent the possible associations between IncRNAs, miRNAs and mRNAs. Red, upregulated; green, downregulated. The red line, the interaction between IncRNAs and miRNAs; the greyish line, the interaction between miRNA and mRNAs.

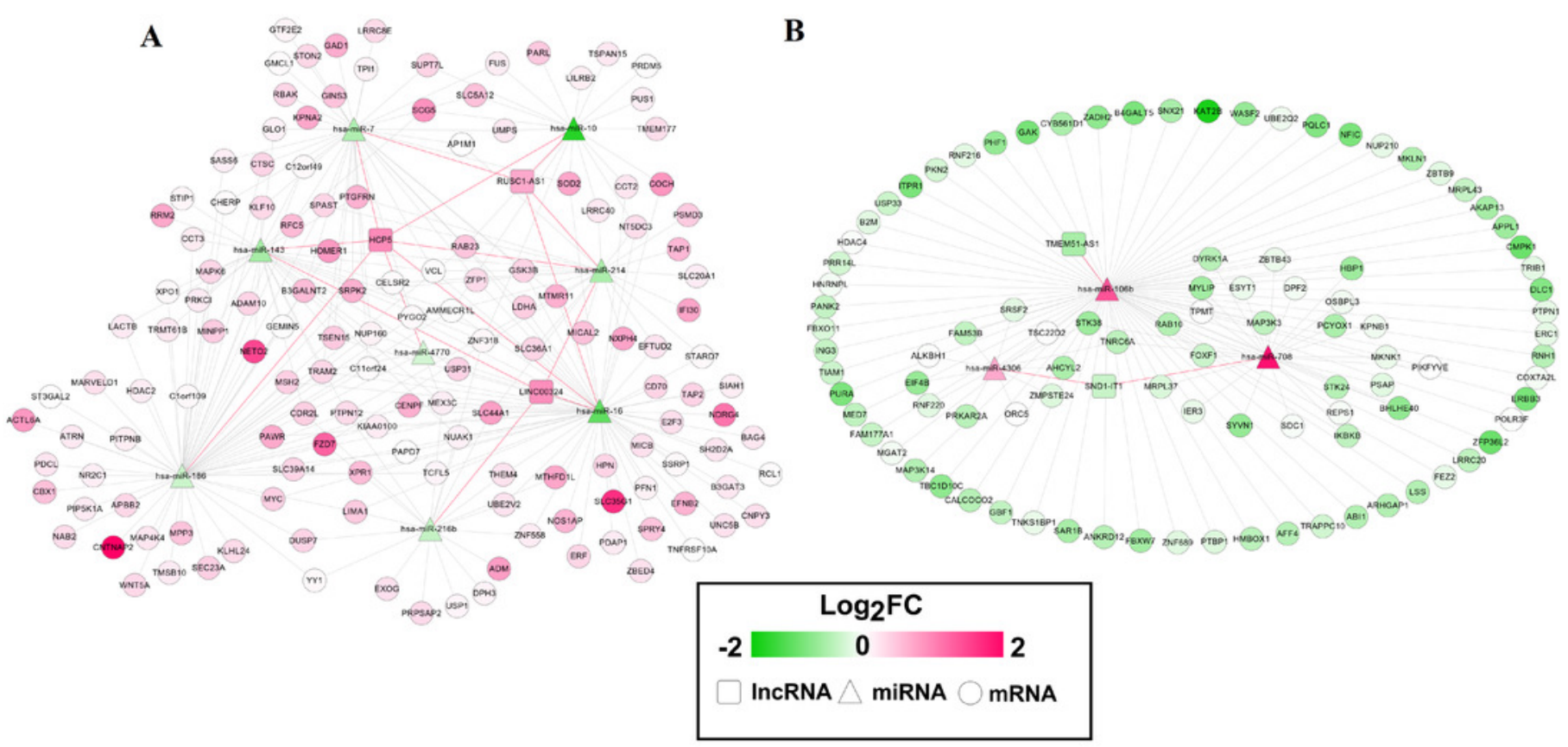




\section{Figure 5}

Prognosis related competing endogenous RNAs (ceRNAs) interaction axes.

A, IncRNA-miRNA-mRNA network for overall survival; B, IncRNA-miRNA-mRNA network for recurrence free survival. Square nodes represent IncRNAs; triangle nodes represent miRNAs; round nodes represent mRNAs. Edges represent the possible associations between IncRNAs, miRNAs and mRNAs. Red, upregulated; green, downregulated.; Kaplan-Meier analysis of the IncRNA (C), miRNA (D) and mRNA (E) of crucial ceRNA axis in which all IncRNA, miRNA and mRNA were prognosis-related and mRNA was an independent prognostic factor.
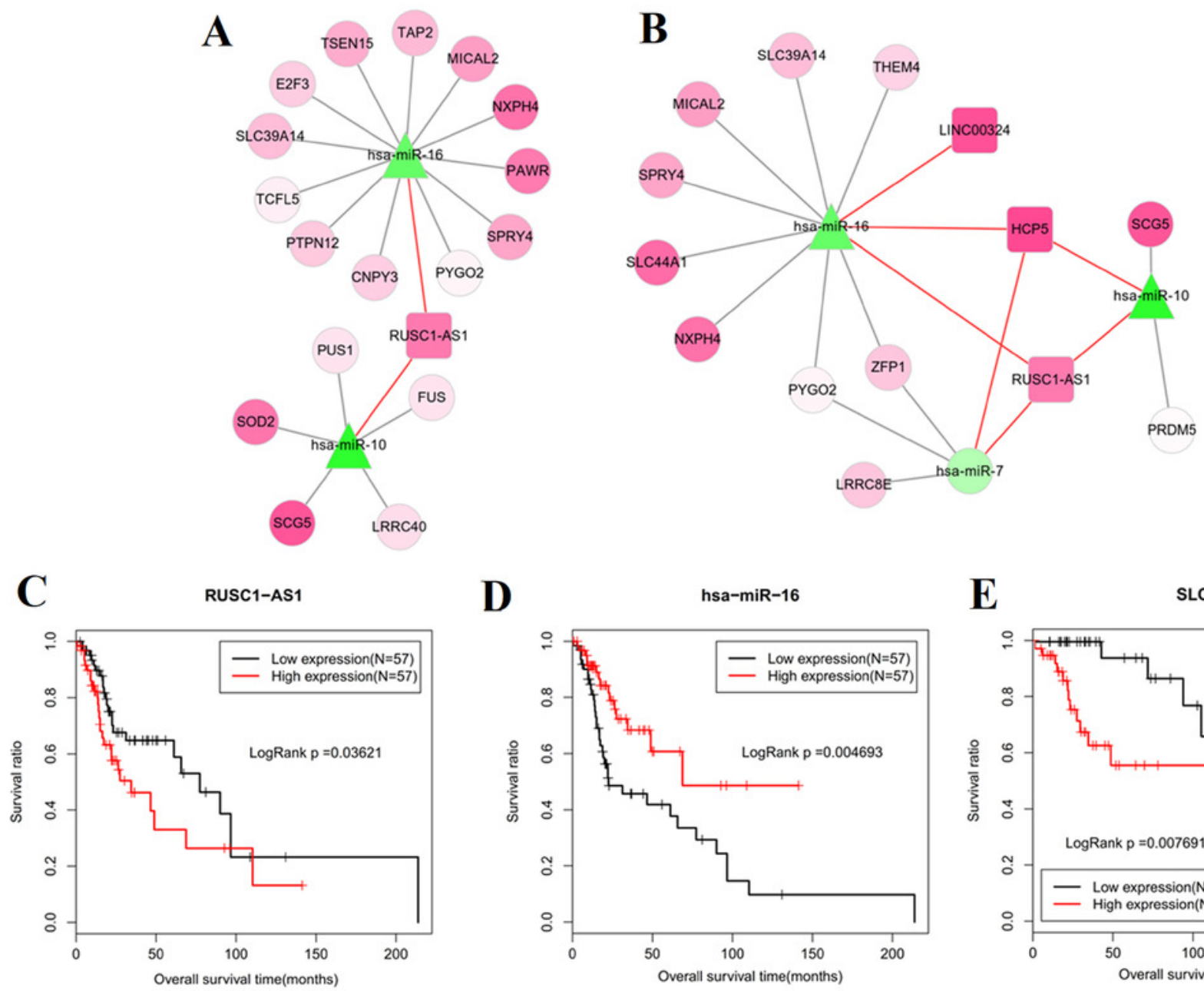

D

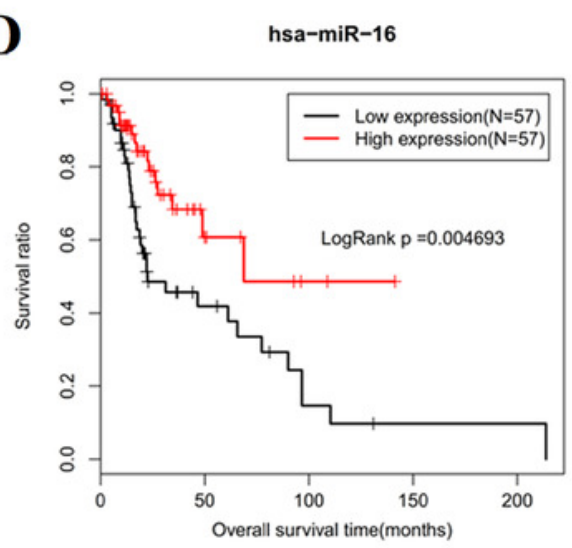

E SLC39A14

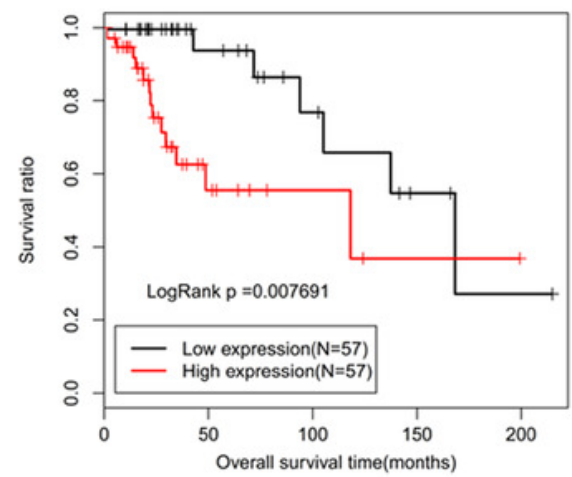


Figure 6

Kaplan-Meier curve of IncRNA TMEM51-AS1 ceRNA related mRNAs.

A, TRAPPC10, which was an independent prognostic factor; B, SNX21, which was overall survival related in univariate Cox regression analysis.
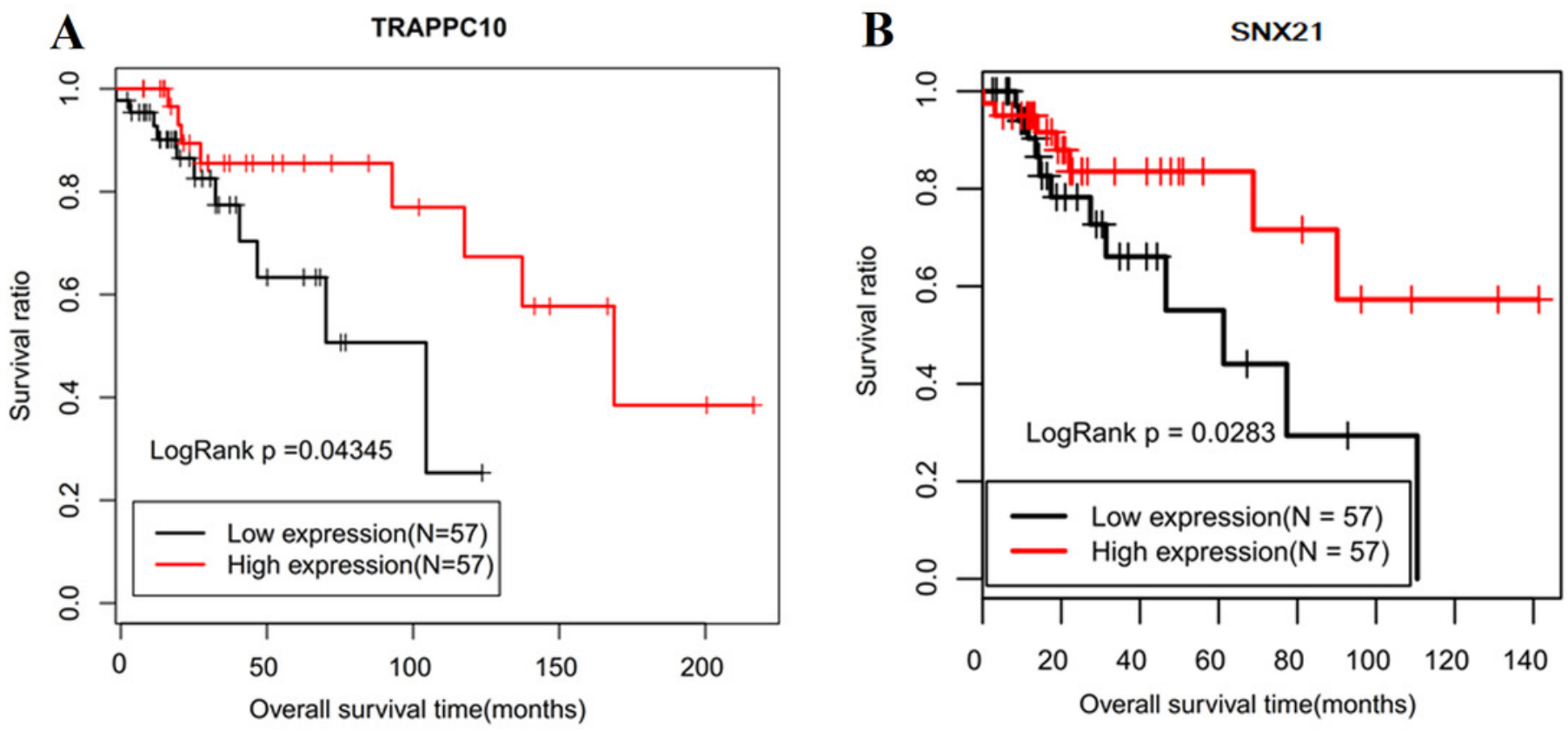


\section{Table $\mathbf{1}$ (on next page)}

Interaction relationship between IncRNA and miRNAs 
1

2 IncRNA

3 HCP5

LINC00324

RUSC1-AS 1

TMEM51-AS1

SND1-IT1
Table 1 Interaction relationship between IncRNA and miRNAs miRNA

hsa-miR-10, hsa-miR-16, hsa-miR-186, hsa-miR-214, hsa-miR-7, hsa-miR-641, hsa-miR-143, hsa-miR-4770, hsa-miR-216b, hsa-miR876

hsa-miR-143, hsa-miR-16, hsa-miR-214, hsa-miR-216b, hsa-miR4770

hsa-miR-214, hsa-miR-10, hsa-miR-16, has-miR-216b, hsa-miR-7

hsa-miR-106b, hsa-miR-765

hsa-miR-708, hsa-miR-4306 


\section{Table 2 (on next page)}

Function enrichment analysis for the genes in ceRNA network 


\begin{tabular}{|c|c|c|c|}
\hline Category & Term & P-value & Genes \\
\hline \multirow[t]{11}{*}{$\begin{array}{l}\text { Biology } \\
\text { process }\end{array}$} & $\begin{array}{l}\text { GO:0006793 phosphorus } \\
\text { metabolic process }\end{array}$ & 0.00122 & $\begin{array}{l}\text { STK38, SLC20A1, ERBB3, NUAK1, MKNK1, ABI1, } \\
\text { PIP5K1A, TRIB1, MAP3K3, SRPK2, MINPP1, } \\
\text { ADAM10, STK24, MSH2, PRKCI, PKN2, PTPN12, } \\
\text { GAK, MAP4K4, MTMR11, MAPK6, GSK3B, DYRK1A, } \\
\text { PTPN1, MAP3K14, ERC1, IKBKB, DUSP7 }\end{array}$ \\
\hline & $\begin{array}{l}\text { GO:0006796 phosphate } \\
\text { metabolic process }\end{array}$ & 0.00122 & $\begin{array}{l}\text { STK38, SLC20A1, ERBB3, NUAK1, MKNK1, ABI1, } \\
\text { PIP5K1A, TRIB1, MAP3K3, SRPK2, MINPP1, } \\
\text { ADAM10, STK24, MSH2, PRKCI, PKN2, PTPN12, } \\
\text { GAK, MAP4K4, MTMR11, MAPK6, GSK3B, DYRK1A, } \\
\text { PTPN1, MAP3K14, ERC1, IKBKB, DUSP7 }\end{array}$ \\
\hline & $\begin{array}{l}\text { GO:0006468 protein amino acid } \\
\text { phosphorylation }\end{array}$ & 0.00498 & $\begin{array}{l}\text { SRPK2, ADAM10, STK38, STK24, NUAK1, ERBB3, } \\
\text { PRKCI, PKN2, MKNK1, ABI1, TRIB1, GAK, MAP4K4, } \\
\text { MAPK6, MAP3K3, GSK3B, DYRK1A, IKBKB, ERC1, } \\
\text { MAP3K14 }\end{array}$ \\
\hline & GO:0016310 phosphorylation & 0.00801 & $\begin{array}{l}\text { SRPK2, ADAM10, STK38, ERBB3, MSH2, STK24, } \\
\text { NUAK1, PRKCI, PKN2, MKNK1, ABI1, PIP5K1A, } \\
\text { TRIB1, GAK, MAP4K4, MAPK6, MAP3K3, GSK3B, } \\
\text { DYRK1A, IKBKB, ERC1, MAP3K14 }\end{array}$ \\
\hline & $\begin{array}{l}\text { GO:0008104 protein } \\
\text { localization }\end{array}$ & 0.01176 & $\begin{array}{l}\text { STON2, SEC23A, XPO1, AP1M1, NUP160, PRKCI, } \\
\text { CENPF, TMSB10, TRAM2, TAP2, GSK3B, NUP210, } \\
\text { TAP1, PIKFYVE, SNX21, RAB23, SCG5, SUPT7L, } \\
\text { SAR1B, RAB10, ERC1, KPNA2, KPNB1 }\end{array}$ \\
\hline & GO:0043407 negative & 0.00991 & STK38, PTPN1, SPRY4, DUSP7 \\
\hline & $\begin{array}{l}\text { regulation of MAP kinase } \\
\text { activity }\end{array}$ & & \\
\hline & $\begin{array}{l}\text { GO:0042981 regulation } \\
\text { apoptosis }\end{array}$ & 0.0165 & $\begin{array}{l}\text { DLC1, DPF2, IER3, ING3, SYVN1, ERBB3, MSH2, } \\
\text { KLF10, PRKCI, AKAP13, CD70, PAWR, SOD2, } \\
\text { TNFRSF10A, BAG4, TIAM1, GSK3B, GLO1, APBB2, } \\
\text { IKBKB, MYC }\end{array}$ \\
\hline & $\begin{array}{l}\text { GO:0043067 regulation } \\
\text { programmed cell death }\end{array}$ & 0.0181 & $\begin{array}{l}\text { DLC1, DPF2, IER3, ING3, SYVN1, ERBB3, MSH2, } \\
\text { KLF10, PRKCI, AKAP13, CD70, PAWR, SOD2, } \\
\text { TNFRSF10A, BAG4, TIAM1, GSK3B, GLO1, APBB2, } \\
\text { IKBKB, MYC }\end{array}$ \\
\hline & GO:0015031 protein transport & 0.0188 & $\begin{array}{l}\text { STON2, SEC23A, XPO1, AP1M1, NUP160, PRKCI, } \\
\text { CENPF, TRAM2, TAP2, GSK3B, NUP210, TAP1, } \\
\text { SNX21, RAB23, SCG5, SAR1B, RAB10, ERC1, KPNA2, } \\
\text { KPNB1 }\end{array}$ \\
\hline & $\begin{array}{l}\text { GO:0010941 regulation of cell } \\
\text { death }\end{array}$ & 0.0188 & $\begin{array}{l}\text { DLC1, DPF2, IER3, ING3, SYVN1, ERBB3, MSH2, } \\
\text { KLF10, PRKCI, AKAP13, CD70, PAWR, SOD2, }\end{array}$ \\
\hline
\end{tabular}




\begin{tabular}{|c|c|c|c|}
\hline & & & $\begin{array}{l}\text { TNFRSF10A, BAG4, TIAM1, GSK3B, GLO1, APBB2, } \\
\text { IKBKB, MYC }\end{array}$ \\
\hline & $\begin{array}{l}\text { GO:0045184 establishment of } \\
\text { protein localization }\end{array}$ & 0.0204 & $\begin{array}{l}\text { STON2, SEC23A, XPO1, AP1M1, NUP160, PRKCI, } \\
\text { CENPF, TRAM2, TAP2, GSK3B, NUP210, TAP1, } \\
\text { SNX21, RAB23, SCG5, SAR1B, RAB10, ERC1, KPNA2, }\end{array}$ \\
\hline & GO:0008219 cell death & 0.0211 & $\begin{array}{l}\text { KPNB1 } \\
\text { FUS, DPF2, DLC1, IER3, MICB, ERBB3, MSH2, } \\
\text { AKAP13, RNF216, PAWR, ITPR1, SOD2, TNFRSF10A, } \\
\text { BAG4, UNC5B, TIAM1, SIAH1, MYC, SPAST }\end{array}$ \\
\hline & $\begin{array}{l}\text { GO:0010033 response } \\
\text { organic substance }\end{array}$ & 0.0217 & $\begin{array}{l}\text { ADAM10, KAT2B, ERBB3, MSH2, KLF10, PRKCI, } \\
\text { CALCOCO2, APPL1, TRIB1, B2M, HDAC4, PRKAR2A, } \\
\text { SDC1, HDAC2, ADM, TAP2, CTSC, PTPN1, MYC }\end{array}$ \\
\hline & GO:0016265 death & 0.0225 & $\begin{array}{l}\text { FUS, DPF2, DLC1, IER3, MICB, ERBB3, MSH2, } \\
\text { AKAP13, RNF216, PAWR, ITPR1, SOD2, TNFRSF10A, } \\
\text { BAG4, UNC5B, TIAM1, SIAH1, MYC, SPAST }\end{array}$ \\
\hline & $\begin{array}{l}\text { GO:0044265 cellular } \\
\text { macromolecule catabolic process }\end{array}$ & 0.0228 & $\begin{array}{l}\text { ADAM10, SYVN1, USP1, RNH1, UBE2V2, RNF216, } \\
\text { MYLIP, UBE2Q2, ZFP36L2, FBXW7, GMCL1, PSMD3, } \\
\text { ZMPSTE24, SIAH1, PCYOX1, USP33, MYC, FBXO11, } \\
\text { USP31 }\end{array}$ \\
\hline & GO:0007049 cell cycle & 0.0405 & $\begin{array}{l}\text { E2F3, KAT2B, MSH2, PAPD7, CENPF, APPL1, GAK, } \\
\text { SASS6, MAPK6, GSK3B, PRDM5, PSMD3, ZNF318, } \\
\text { HBP1, SIAH1, APBB2, KPNA2, MYC, SPAST }\end{array}$ \\
\hline & $\begin{array}{l}\text { GO:0009057 macromolecule } \\
\text { catabolic process }\end{array}$ & 0.0427 & $\begin{array}{l}\text { ADAM10, SYVN1, USP1, RNH1, UBE2V2, RNF216, } \\
\text { MYLIP, UBE2Q2, ZFP36L2, FBXW7, GMCL1, PSMD3, } \\
\text { ZMPSTE24, SIAH1, PCYOX1, USP33, MYC, FBXO11, } \\
\text { USP31 }\end{array}$ \\
\hline \multirow{6}{*}{$\begin{array}{l}\text { KEGG } \\
\text { pathway }\end{array}$} & hsa05210:Colorectal cancer & 0.0377 & MSH2, GSK3B, APPL1, MYC, FZD7 \\
\hline & hsa04210:Apoptosis & 0.0421 & TNFRSF10A, PRKAR2A, EXOG, IKBKB, MAP3K14 \\
\hline & $\begin{array}{l}\text { hsa00562:Inositol phosphate } \\
\text { metabolism }\end{array}$ & 0.0477 & MINPP1, TPI1, PIKFYVE, PIP5K1A \\
\hline & $\begin{array}{l}\text { hsa05169:Epstein-Barr virus } \\
\text { infection }\end{array}$ & 0.00175 & $\begin{array}{l}\text { POLR3F, XPO1, HDAC4, GTF2E2, HDAC2, GSK3B, } \\
\text { PSMD3, MAP3K14, IKBKB, MYC }\end{array}$ \\
\hline & hsa05166:HTLV-I infection & 0.0122 & $\begin{array}{l}\text { WNT5A, XPO1, E2F3, KAT2B, MAP3K3, GSK3B, } \\
\text { MAP3K14, IKBKB, MYC, FZD7 }\end{array}$ \\
\hline & $\begin{array}{l}\text { hsa05205:Proteoglycans } \\
\text { cancer }\end{array}$ & 0.0267 & $\begin{array}{l}\text { WNT5A, EIF4B, SDC1, TIAM1, ERBB3, MYC, FZD7, } \\
\text { ITPR1 }\end{array}$ \\
\hline
\end{tabular}




\section{Table 3(on next page)}

Prognosis related IncRNAs, miRNAs and mRNAs in ceRNA network 
Table 3 Prognosis related IncRNAs, miRNAs and mRNAs in ceRNA network

\begin{tabular}{|c|c|c|c|c|c|c|c|}
\hline \multicolumn{4}{|c|}{ Overall survival } & \multicolumn{4}{|c|}{ Recurrence free survival } \\
\hline & RNA & $\exp ($ coef $)$ & $\mathrm{p}$ & & RNA & $\exp (\operatorname{coef})$ & $\mathrm{p}$ \\
\hline \multirow[t]{2}{*}{ miRNA } & hsa-miR-16 & 0.506 & 0.00048 & miRNA & hsa-miR-10 & 0.678 & 0.0135 \\
\hline & hsa-miR-10 & 0.653 & 0.016 & & hsa-miR-16 & 0.523 & 0.00355 \\
\hline $\operatorname{lncRNA}$ & RUSC1-AS1 & 1.09 & 0.01 & & hsa-miR-7 & 1.75 & 0.011 \\
\hline \multirow[t]{29}{*}{ mRNA } & ADAM10 & 2.01 & 0.0435 & $\operatorname{lncRNA}$ & LINC00324 & 1.38 & 0.0205 \\
\hline & AHCYL2 & 0.739 & 0.0315 & mRNA & AFF4 & 2.43 & 0.036 \\
\hline & CNPY3 & 0.602 & 0.048 & & CELSR2 & 0.576 & 0.041 \\
\hline & DLC1 & 1.56 & 0.025 & & ERBB3 & 0.392 & 0.041 \\
\hline & E2F3 & 0.661 & 0.0355 & & LRRC8E & 0.577 & 0.029 \\
\hline & FUS & 0.531 & 0.0345 & & MAP4K4 & 2.12 & 0.0475 \\
\hline & $\mathrm{HPN}$ & 0.878 & 0.036 & & MICAL2 & 1.76 & 0.0115 \\
\hline & ITPR1 & 2.19 & 0.036 & & NXPH4 & 0.702 & 0.0265 \\
\hline & LRRC40 & 2.56 & 0.042 & & PCYOX1 & 0.484 & 0.0445 \\
\hline & MICAL2 & 1.49 & 0.0325 & & PRDM5 & 1.64 & 0.0135 \\
\hline & NXPH4 & 0.722 & 0.032 & & PTBP1 & 8.8 & 0.047 \\
\hline & PAWR & 1.57 & 0.037 & & PTPN1 & 3.26 & 0.038 \\
\hline & PRPSAP2 & 0.563 & 0.045 & & PYGO2 & 0.252 & 0.036 \\
\hline & PTPN12 & 2.19 & 0.0485 & & RRM2 & 2.32 & 0.043 \\
\hline & PUS1 & 0.668 & 0.0385 & & SCG5 & 1.84 & 0.00065 \\
\hline & PYGO2 & 0.334 & 0.0485 & & $\mathrm{SDC1}$ & 0.459 & 0.042 \\
\hline & RAB10 & 1.59 & 0.0295 & & SLC39A14 & 1.85 & 0.042 \\
\hline & SAR1B & 1.62 & 0.039 & & SLC44A1 & 0.445 & 0.011 \\
\hline & SCG5 & 1.4 & 0.0295 & & SPRY4 & 2.28 & 0.007 \\
\hline & SLC39A14 & 1.78 & 0.027 & & ST3GAL2 & 2.34 & 0.033 \\
\hline & SNX21 & 0.502 & 0.048 & & THEM4 & 0.278 & 0.006 \\
\hline & SOD2 & 0.736 & 0.038 & & ZFP1 & 3.35 & 0.032 \\
\hline & SPRY4 & 1.98 & 0.023 & & & & \\
\hline & ST3GAL2 & 1.87 & 0.0345 & & & & \\
\hline & TAP2 & 0.68 & 0.0425 & & & & \\
\hline & TCFL5 & 0.613 & 0.033 & & & & \\
\hline & TRAPPC10 & 0.324 & 0.037 & & & & \\
\hline & TSC22D2 & 1.36 & 0.0295 & & & & \\
\hline & TSEN15 & 1.58 & 0.0415 & & & & \\
\hline
\end{tabular}

2

3 
Table 4 (on next page)

Independent prognostic factors for LSCC by multivariate Cox regression 


\begin{tabular}{|c|c|c|c|c|c|c|c|c|c|}
\hline \multicolumn{5}{|c|}{ OS } & \multicolumn{5}{|c|}{ RFS } \\
\hline \multirow[t]{3}{*}{ ID } & \multirow[t]{3}{*}{ P-value } & \multirow[t]{3}{*}{ HR } & \multicolumn{2}{|c|}{$95 \% \mathrm{CI}$} & \multirow[t]{3}{*}{ ID } & \multirow[t]{3}{*}{ P-value } & \multirow[t]{3}{*}{ HR } & \multicolumn{2}{|c|}{$95 \% \mathrm{CI}$} \\
\hline & & & Lower & Upper & & & & Lower & Upper \\
\hline & & & limit & limit & & & & limit & limit \\
\hline TRAPPC10 & 0.0106 & 0.0941 & 0.01535 & 0.5768 & SLC44A1 & 0.0055 & 0.1719 & 0.0496 & 0.5958 \\
\hline Alcohol & 0.0252 & 0.0391 & 0.00229 & 0.668 & THEM4 & 0.0101 & 0.2215 & 0.07023 & 0.6988 \\
\hline SLC39A14 & 0.0289 & 9.37 & 1.26 & 69.8 & RRM2 & 0.0151 & 34.8562 & 1.99009 & 610.503 \\
\hline SOD2 & 0.0456 & 2.748 & 1.01992 & 7.4038 & Age & 0.0875 & 0.06502 & 0.00283 & 1.494 \\
\hline SCG5 & 0.0515 & 8.6 & 0.986 & 75.1 & $\mathrm{~T}$ & 0.1882 & 0.09721 & 0.00302 & 3.129 \\
\hline Grade & 0.0536 & 0.0053 & 0.000025 & 1.09 & Stage & 0.1883 & 14.2194 & 0.27247 & 742.056 \\
\hline MICAL2 & 0.0872 & 0.141 & 0.0149 & 1.33 & ZFP1 & 0.2108 & 3.5709 & 0.48649 & 26.2105 \\
\hline RUSC1-AS1 & 0.0894 & 1.1434 & 0.97957 & 1.3347 & LINC00324 & 0.2134 & 1.5429 & 0.77921 & 3.0549 \\
\hline Gender & 0.1057 & 0.002 & $1.08 \mathrm{E}-06$ & 3.72 & PCYOX1 & 0.2206 & 0.16586 & 0.009362 & 2.939 \\
\hline CNPY3 & 0.1069 & 43.1 & 0.444 & 4170 & CELSR2 & 0.2233 & 0.12475 & 0.004376 & 3.556 \\
\hline PUS1 & 0.1534 & 0.0927 & 0.00354 & 2.43 & NXPH4 & 0.2429 & 0.58688 & 0.239935 & 1.435 \\
\hline HPN & 0.1538 & 0.398 & 0.112 & 1.41 & Gender & 0.2507 & 0.11327 & 0.002754 & 4.658 \\
\hline hsa-mir-16-2 & 0.1626 & 0.5574 & 0.24539 & 1.266 & PTPN1 & 0.2567 & 17.1232 & 0.126445 & 2318.83 \\
\hline Age & 0.1641 & 0.0323 & 0.000256 & 4.07 & AFF4 & 0.3093 & 17.52882 & 0.070156 & 4379.665 \\
\hline PTPN12 & 0.204 & 42.7 & 0.13 & 14000 & PYGO2 & 0.3218 & 0.06118 & 0.000243 & 15.379 \\
\hline ADAM10 & 0.245 & 0.136 & 0.00472 & 3.93 & SLC39A14 & 0.3303 & 1.5919 & 0.62436 & 4.0587 \\
\hline LRRC40 & 0.2467 & 0.0333 & 0.000106 & 10.5 & SPRY4 & 0.3574 & 1.6716 & 0.55973 & 4.9922 \\
\hline Stage & 0.2589 & 185 & 0.0215 & 158000 & Grade & 0.4953 & 0.24206 & 0.004104 & 14.277 \\
\hline RAB10 & 0.2779 & 0.0066 & $7.71 \mathrm{E}-07$ & 57.1 & MAP4K4 & 0.4973 & 0.21996 & 0.002775 & 17.436 \\
\hline $\mathrm{T}$ & 0.2814 & 0.0214 & 0.000020 & 23.3 & $\begin{array}{l}\text { hsa-mir-16- } \\
2\end{array}$ & 0.50105 & 0.7001 & 0.24778 & 1.978 \\
\hline TSC22D2 & 0.3088 & 1.7322 & 0.60129 & 4.99 & ERBB3 & 0.6193 & 2.56113 & 0.06268 & 104.645 \\
\hline FUS & 0.3262 & 0.0128 & $2.11 \mathrm{E}-06$ & 77.1 & PTBP1 & 0.629 & 8.46884 & 0.00146 & 49149.03 \\
\hline DLC1 & 0.3279 & 0.168 & 0.00472 & 5.99 & tobacco & 0.6901 & 0.59073 & 0.04442 & 7.856 \\
\hline PRPSAP2 & 0.328 & 0.0598 & 0.000212 & 16.9 & SCG5 & 0.7040 & 1.2036 & 0.46263 & 3.1314 \\
\hline TSEN15 & 0.3295 & 2.1853 & 0.45397 & 10.5197 & PRDM5 & 0.7108 & 1.45392 & 0.20106 & 10.514 \\
\hline ST3GAL2 & 0.3643 & 1.4916 & 0.62882 & 3.5381 & $\mathrm{~N}$ & 0.7903 & 0.69963 & 0.05030 & 9.731 \\
\hline PYGO2 & 0.4608 & 6.86 & 0.0412 & 1140 & LRRC8E & 0.7914 & 0.70897 & 0.05545 & 9.065 \\
\hline E2F3 & 0.4736 & 2.69 & 0.18 & 40.2 & MICAL2 & 0.8244 & 1.2676 & 0.15616 & 10.289 \\
\hline $\mathrm{N}$ & 0.5037 & 0.203 & 0.00188 & 21.8 & SDC1 & 0.8476 & 1.1355 & 0.31079 & 4.1489 \\
\hline SPRY4 & 0.5551 & 1.3026 & 0.54132 & 3.1346 & hsa-mir-7-2 & 0.8556 & 1.1305 & 0.30175 & 4.2351 \\
\hline TAP2 & 0.574 & 0.7112 & 0.21679 & 2.3332 & hsa-mir-10a & 0.9186 & 0.9692 & 0.53189 & 1.7661 \\
\hline NXPH4 & 0.6486 & 0.784 & 0.276 & 2.23 & ST3GAL2 & 0.9385 & 0.9482 & 0.24524 & 3.666 \\
\hline TCFL5 & 0.6954 & 0.7436 & 0.16866 & 3.278 & Alcohol & 0.9937 & 1.01803 & 0.01187 & 87.316 \\
\hline Tobacco & 0.717 & 1.67 & 0.105 & 26.5 & & & & & \\
\hline SNX21 & 0.8253 & 0.8703 & 0.25359 & 2.9871 & & & & & \\
\hline
\end{tabular}




\begin{tabular}{lllll}
\hline hsa-mir-10a & 0.8392 & 0.9451 & 0.54739 & 1.6316 \\
PAWR & 0.842 & 1.7 & 0.00918 & 315 \\
SAR1B & 0.9106 & 1.25 & 0.0244 & 64.3 \\
ITPR1 & 0.9121 & 1.19 & 0.0539 & 26.3 \\
AHCYL2 & 0.9767 & 1.06 & 0.0236 & 47.5 \\
\hline
\end{tabular}

2

3 


\section{Table 5 (on next page)}

Clinical characteristics related IncRNAs, miRNAs and mRNAs in prognostic ceRNA network

Genes with underline were recurrence free survival related; the other genes were overall survival related. Gene in bold was both recurrence free and overall survival related. 


\begin{tabular}{|c|c|c|c|}
\hline \multirow{2}{*}{ Clinical characteristics } & \multicolumn{3}{|r|}{ Significant related } \\
\hline & $\operatorname{lncRNA}$ & miRNA & mRNA \\
\hline $\operatorname{Age}(\geq 60 /<60$ y) & - & - & ADAM10, FUS, MICAL2, LRRC8E \\
\hline Gender(Male/Female) & - & - & $\begin{array}{c}\text { DLC1, HPN, PTPN12, SNX21, ST3GAL2, LRRC8E, NXPH4, } \\
\underline{\text { PTPN1, SCG5, }}\end{array}$ \\
\hline Alcohol use(Yes/No) & - & - & CNPY3, PYGO2, SLC39A14, TAP2 \\
\hline Pathologic_M(M0/-) & - & - & $\begin{array}{c}\text { ADAM10, CNPY3, E2F3, SPRY4, LRRC8E, MAP4K4, PTBP1, } \\
\underline{\text { RRM2 }}\end{array}$ \\
\hline Pathologic_N(N0/N1/N2/N3/-) & RUSC1-AS1 & - & $\begin{array}{c}\text { DLC1, FUS, MICAL2, SOD2, } \underline{\text { PCYOX1, RRM2, SLC39A14, }} \\
\underline{\text { ZFP1 }}\end{array}$ \\
\hline Pathologic_T(T1/T2/T3/T4/-) & - & - & LRRC40, PRPSAP2, SOD2, TSEN15, CELSR2, PTPN1 \\
\hline Pathologic_stage(I/II/III/IV/-) & - & - & $\begin{array}{c}\text { MICAL2, PRPSAP2, SPRY4, } \underline{\text { CELSR2, MAP4K4, PCYOX1, }} \\
\underline{\text { PTPN1 }}\end{array}$ \\
\hline Grade(G1/G2/G3/G4) & - & - & AHCYL2, DLC1, PCYOX1, PTPN1, RRM2 \\
\hline Tobacco use(Reform/Current/Never) & - & - & HPN, RAB10, SAR1B, SOD2, TAP2 \\
\hline
\end{tabular}

3 Genes with underline were recurrence free survival related; the other genes were overall survival related. Gene in bold was both 4 recurrence free and overall survival related.

5 